\title{
Equações de Diferença \\ Matricial Linear: \\ Soluções e a \\ Transformada de Laurent
}

Elisa Yoshiko Takada

DISSERTAÇÃO APRESENTADA

$\mathrm{AO}$

INSTITUTO DE MATEMÁTICA E ESTATÍSTICA

DA

UNIVERSIDADE DE SÃO PAULO

PARA OBTENÇÃO DO GRAU DE MESTRE

EM

MATEMÁTICA APLICADA

Área de Concentração: Análise Numérica

Orientador: Prof. Dr. Cyro de Carvalho Patarra

-São Paulo, Maio de 1993- 
- i -

A meu pai, Hideo, Com muitas saudades. 


\section{Agradecimentos}

- Agradeço ao meu orientador, Cyro Patarra, sem o qual esta dissertação não teria sido realizada, por mostrar-me que a Matemática Aplicada pode ser muito mais fascinante do que parece.

- Agradeço ao Fernando, pela sua atenção, seu carinho e paciência com que reviu os originais deste trabalho, sempre indicando o melhor caminho a seguir (nem sempre menos árduo).

- Agradeço a todos da CPG, pelo carinho, atenção e pela força que sempre me deram.

Gostaria de agradecer a todos os meus amigos e amigas que, direta ou indiretamente, apoiaram-me nos muitos momentos difíceis. Em especial,

- ao Jorge, pela amizade, pelo exemplo e pelo desprendimento com que sempre me ajudou,

- à Olga, pelas longas (e proveitosas) conversas que tivemos,

- à Roseli e à Rosa, pelo carinho e apoio.

- Agradeço a meus pais, que, com carinho, sempre me incentivaram a estudar. 


\section{Resumo}

Neste trabalho, fazemos uma extensão da teoria das equações de diferença lineares para o caso matricial, mostrando que as soluções, seqüências de matrizes, podem ser obtidas nos moldes do caso escalar através dos zeros de um polinômio matricial, chamados de solventes. Como a existência desses solventes não é uma condição suficiente para a existência de um conjunto fundamental de soluções, apresentamos alguns métodos alternativos para a obtenção dessas soluções baseados na noção de cadeia de solventes e através da aplicação da transformada de Laurent.

\section{Abstract}

In this work, we make an extension on the theory of llinear difference equations to the matricial case showing thar their solutions, sequences of matrices, can be obtained by the roots of matricial polynomials, called solvents. As the existence of these solvents is not a sufficient condition to the existence of a fundamental solution set for a matricial linear homogeneous difference equation, we present some alternative methods to find an analytic solution, by the Laurent transform and by a chain of solvents. 


\section{Introdução}

Uma base do subespaço de soluções de uma equação de diferença linear (escalar) de ordem $\mathrm{n}$ a coeficientes constantes pode ser facilmente encontrada através das raízes do polinômio característico associado. Ao estendermos a teoria das equações de diferença lineares ao caso matricial, temos seqüências de matrizes como suas soluções.

A primeira tentativa no sentido de obter essas soluções é associar à equação de diferença matricial linear um polinômio matricial e, através de suas raízes, construir uma base, como é feito usualmente no caso escalar. Aqui deparamo-nos com dois problemas. O primeiro: é difícil garantir a existência de raízes de um polinômio matricial já que não temos um Teorema Fundamental da Álgebra para esse tipo de polinômios, além do fato de que, mesmo que essas rauzes existam, é difícil determiná-las. O segundo: não existe base para o conjunto de soluções de uma equação de diferença matricial linear no sentido usual de base de um espaço vetorial.

Em nosso trabalho, não preocupamo-nos em obter os solventes. Existem vários autores lidando com esse problema, por exemplo, Dennis [2], Gohberg [5], Jódar [8], entre outros. Fernando Menzaque, em sua tese de doutorado [17] apresenta algoritmos que determinam todos os solventes de um polinômio matricial cujos coeficientes são simultaneamente triangularizáveis.

O nosso objetivo é resolver um problema de valores iniciais: obter uma solução de uma equação de diferença linear matricial mediante condições iniciais dadas. Procuramos fazêlo de várias formas: a primeira delas, impondo condições sobre as seqüências de matrizes geradas por solventes para que estas formem um conjunto fundamental de soluções. A segunda forma faz uso de uma cadeia de solventes. A terceira forma, consiste em modificar o problema, transformando a equação de diferença em uma equação algébrica através da aplicação da transformada de Laurent, ou z-transformada como é conhecida, adaptada à aplicação para o caso matricial. Assim, obtemos uma solução analítica fechada sem a necessidade dos solventes. 
A técnica das transformadas é muito utilizada na teoria das equações diferenciais, sendo a de Laplace a mais conhecida. Várias transformadas foram desenvolvidas especialmente para serem aplicadas em sistemas discretos, como a de Laplace discreta, Dirichlet, Laurent, etc. Em nosso trabalho, dedicamo-nos à transformada de Laurent, pois a sua aplicação em equações de diferença matriciais gera as lambda-matrizes, cuja teoria traz resultados interessantes.

No Capítulo 1, apresentamos a teoria das equações de diferença matricial lineares a coeficientes constantes de ordem n, onde destacamos o Teorema de Existência e Unicidade de soluções dessa equação e a definição de conjunto fundamental de soluções.

No Capítulo 2 estão os requisitos necessários aos capítulos subsequentes. Nele, apresentamos rapidamente os polinômios matriciais, a matriz de Vandermonde generalizada e as lambda-matrizes. Os dois primeiros itens serão utilizados na construção de um conjunto fundamental de soluçōes, e o terceiro item será utilizado na aplicação da transformada de Laurent a uma equação de diferença matricial linear.

No Capítulo 3, mostramos em que condições podemos obter um conjunto fundamental de soluções e no Capítulo 4, baseado em um artigo de Hernández [7], apresentamos uma solução analítica fechada de uma equação de diferença matricial linear através de uma cadeia de solventes.

Finalmente, no Capítulo 5 apresentamos a transformada de Laurent matricial e, através da aplicação dessa transformada em uma equação de diferença matricial, obtemos uma solução dessa equação. Neste Capítulo, são necessários alguns resultados da teoria de Análise Complexa. 


\section{Conteúdo}

1 Equação de Diferença 1

1.1 Equação de Diferença Matricial Linear . . . . . . . . . . . . . . 2

2 Lambda-matriz e Polinômio Matricial 13

2.1 Lambda-matriz . . . . . . . . . . . . . . . . . . . . . . 13

2.2 Polinômio matricial . . . . . . . . . . . . . . . . . . . . . . . . . . . . . . . .

2.3 Matriz de Vandermonde por blocos . . . . . . . . . . . . . . . 19

3 Conjunto Fundamental de Soluções 23

3.1 Método clássico . . . . . . . . . . . . . . . . . . . . 23

3.2 Conjunto fundamental de soluções . . . . . . . . . . . . . . . . 24

4 Cadeia de solventes 33

4.1 Cadeia de Solventes . . . . . . . . . . . . . . . . . 33

5 Transformada de Laurent matricial $\quad 47$

5.1 Transformada de Laurent - Caso Escalar . . . . . . . . . . . . . . . 47

5.2 Transformada de Laurent - Caso matricial . . . . . . . . . . . . . . . 59

5.3 A Transformada e a Solução . . . . . . . . . . . . . . . . . . 63

5.4 Decomposição em frações parciais . . . . . . . . . . . . . . . 65

5.5 Aplicação . . . . . . . . . . . . . . . . . . . . . 67

5.6 Exemplo . . . . . . . . . . . . . . . . . . . 70

5.7 Conclusâo . . . . . . . . . . . . . . . . . . 71

$\begin{array}{ll}\text { Bibliografia } & 73\end{array}$ 


\section{Capítulo 1}

\section{EQUAÇÃO DE DIFERENÇA LINEAR MATRICIAL}

As equações de diferença ou relações de recorrência aparecem em vários contextos. Sua aplicação ocorre em modelagem matemática, em probabilidade e, entre outros exemplos, podem ser usados como aproximação às equações diferenciais. Várias aplicaçôes podem ser encontradas em [6] e [11].

As equações de diferença matricial podem ser obtidas a partir de alguns tipos de discretização das equações diferenciais parciais não lineares, como as que descrevem as equações de previsão do tempo ou a equação da chama. Como vários problemas são formulados com um tipo especial de equações de diferença, a saber, as lineares, vamos dedicar nosso trabalho ao estudo dessas equações.

Neste Capítulo, apresentamos a teoria das equaçôes de diferença matricial linear, onde destacamos o Teorema de Existência e Unicidade de soluçâo dessas equações e o fato de um conjunto fundamental de soluções não ser uma base do espaço de soluções. 


\subsection{Equação de Diferença Linear: Caso Matricial}

Nesta seção, fazemos uma extensão das equações de diferença lineares considerando os coeficientes e as variáveis de uma equação de diferença linear como matrizes quadradas e desenvolvemos a teoria à semelhança do que é feito para as equações de diferença linear escalares. Denotaremos uma matriz por blocos com seus elementos entre colchetes, indicando-a com letra maiúscula.

Seja $\mathbb{C}^{p \times q}$ o conjunto das matrizes de $p$ linhas e $q$ colunas com elementos em $\mathbb{C}$. Seja $\mathcal{M}=\left\{\left\{Y_{k}\right\}_{k=0}^{\infty}, Y_{k} \in \mathbb{C}^{m \times m}\right\}$ o espaço vetorial das seqüências de matrizes de $\mathbb{C}^{m \times m}$. Escreveremos $Y=\left\{Y_{k}\right\}_{k=0}^{\infty}$ ou simplesmente $Y=\left\{Y_{k}\right\}$ para indicar a seqüência $Y \in \mathcal{M}$.

Definição 1.1 : Uma equação de diferença matricial de ordem n é uma relação da forma :

$$
Y_{k+n}=F\left(k, Y_{k}, Y_{k+1}, \ldots, Y_{k+n-1}\right)=0, \quad k=0,1, \ldots
$$

A expressão acima representa não só uma equação, mas um sistema infinito de equações, ou seja, cada valor de $k$ está associada a uma equação.

Definição 1.2 : Uma seqüência $\left\{Y_{k}\right\} \in \mathcal{M}$ que satisfaz a equação acima é chamada solução da equação de diferença.

Consideremos o seguinte caso especial :

Definição 1.3 : A equação

$$
C_{0, k} Y_{k+n}+\cdots+C_{n, k} Y_{k}=G_{k}, \quad k=0,1, \ldots,
$$

$\operatorname{com}\left\{C_{i, k}\right\}_{k=0}^{\infty},\left\{Y_{k}\right\},\left\{G_{k}\right\} \in \mathcal{M}$, é chamada equação de diferença matricial linear. Se $C_{0, k}$ e $C_{n, k}$ são não nulas para $k=0,1, \ldots$, dizemos que a ordem da equação é $n$. 
Observamos que cada elemento das seqüências $\left\{C_{i, k}\right\}_{k=0}^{\infty},\left\{Y_{k}\right\}$ e $\left\{G_{k}\right\}$ podem ser vistos como operadores lineares em $\mathbb{C}^{m \times m}$. Em nosso trabalho, abordamos o caso em que a seqüência de matrizes $\left\{C_{i, k}\right\}_{k=0}^{\infty}$ é constante, ou seja, independe de $k$ sendo, por isso, denotadas por $C_{i}$. Nesse caso, temos a seguinte equação :

$$
C_{0} Y_{k+n}+C_{1} Y_{k+n-1}+\cdots+C_{n} Y_{k}=G_{k}, \quad k=0,1, \ldots
$$

Se $C_{0}$ é não singular, a equação acima pode ser escrita sem perda de generalidade como :

$$
Y_{k+n}+A_{1} Y_{k+n-1}+\cdots+A_{n} Y_{k}=B_{k}, \quad k=0,1, \ldots
$$

Definição 1.4 : Se $B_{k}=0$ para todo $k$, a equação

$$
Y_{k+n}+A_{1} Y_{k+n-1}+\cdots+A_{n} Y_{k}=0, \quad k=0,1, \ldots,
$$

é chamada equaçâo de diferença matricial linear homogênea associada a (1.1).

Observação 1.1 : Chamamos de equação de diferença matricial linear à esquerda à expressão abaixo :

$$
Y_{k+n} C_{0}+Y_{k+n-1} C_{1}+\cdots+Y_{k} C_{n}=B_{k}, \quad k=0,1, \ldots .
$$

As equações (1.1) e (1.2) apresentam propriedades semelhantes pois a segunda pode ser obtida através da transposição das matrizes em (1.1). Por isso, vamos centrar nossa atençâo somente na primeira equação.

O objetivo de nosso trabalho é obter soluções para o seguinte problema de valores iniciais ou problema de Cauchy:

Problema de Cauchy : Dados $D_{0}, \ldots, D_{n-1} \in \mathbb{C}^{m \times m}$, determinar uma solução $\left\{Y_{k}\right\} \in \mathcal{M}$ da equação (1.1) que satisfaça as seguintes condições iniciais : $Y_{0}=D_{0}, \ldots, Y_{n-1}=D_{n-1}$. 
Apresentamos, então, o teorema que garante a existência e unicidade de soluçôes do problema de valores iniciais acima.

\section{Teorema 1.1 (Teorema de Existência e Unicidade)}

Dadas as matrizes $D_{0}, \ldots, D_{n-1} e m \mathbb{C}^{m \times m}$, a equação de diferença de ordem $n$

$$
Y_{k+n}+A_{1} Y_{k+n-1}+\cdots+A_{n} Y_{k}=B_{k}, \quad k=0,1, \ldots,
$$

admite uma única soluçâo $\left\{Y_{k}\right\} \in \mathcal{M}$ que satisfaz $n$ condições iniciais : $Y_{0}=$ $D_{0}, \ldots, Y_{n-1}=D_{n-1}$.

Demonstração : Usando as condições iniciais, a solução de (1.3) pode ser obtida recursivamente. Escrevendo

$$
Y_{k+n}=B_{k}-A_{1} Y_{k+n-1}-\cdots-A_{n} Y_{k},
$$

fazemos $k=0,1, \ldots$ para obter os elementos $Y_{n}, Y_{n+1}, \ldots$, respectivamente. Para mostrar a unicidade da solução, consideramos $X=\left\{X_{k}\right\} \in \mathcal{M}, Z=\left\{Z_{k}\right\} \in \mathcal{M}$ duas soluçòes de (1.3) que satisfazem as condiçòes iniciais :

$$
X_{k}=Z_{k}=D_{k}, \quad k=0, \ldots, n-1 .
$$

Seja $W=X-Z \in \mathcal{M}$. Observamos que $W$ é uma solução da equação homogênea associada a (1.3),

$$
W_{k+n}=-A_{1} W_{k+n-1}-\cdots-A_{n} W_{k}, \quad k=0,1, \ldots,
$$

e que satisfaz as seguintes condições iniciais : $W_{0}=\cdots=W_{n-1}=0$.

Mostremos que $W=0$. Para $k=0$ em (1.4), obtemos $W_{n}=0$. Supondo que, para $j>0, W_{j}=\cdots=W_{j+n-1}=0$, temos $W_{j+n}=-A_{1} W_{j+n-1}-\cdots-A_{n} W_{j}=0$. Portanto, segue por indução que $W=0$, ou seja, $X=Z$.

Corolário 1.1 : Se $\left\{Y_{k}\right\} \in \mathcal{M}$ é uma soluçấo da equação homogênea associada à equação (1.3) que se anula em $k=0,1, \ldots, n-1$, então $\left\{Y_{k}\right\}$ é a solução nula. 
Observamos que, conhecidas $n$ condições iniciais consecutivas $D_{s}, \ldots, D_{s+n-1}$ $\in \mathbb{C}^{m \times m}$ com $s \in \mathbb{Z}_{+}, s \neq 0$, podemos garantir a existência e unicidade da solução de (1.3) adicionando a hipótese de que $A_{n}$ é não-singular.

Podemos obter explicitamente uma solução de uma equação de diferença matricial linear de ordem $n$ através de uma mudança de variável. Dessa forma, reduzimos a equação de ordem $n$ a um sistema de equações de diferença lineares de ordem 1 que pode ser resolvido recursivamente. De fato, seja :

$$
Y_{k+n}+A_{1} Y_{k+n-1}+\cdots+A_{n} Y_{k}=B_{k}, \quad k=0,1, \ldots
$$

Fazendo $Z_{k}^{(r)}=Y_{k+r-1}, \quad r=1, \ldots, n$, temos

$$
\left\{\begin{aligned}
Z_{k+1}^{(1)} & =Y_{k+1} \\
Z_{k+1}^{(2)} & =Y_{k+2} \\
& \vdots \\
Z_{k+1}^{(n)} & =Y_{k+n}=B_{k}-A_{1} Z_{k}^{(n)}-A_{2} Z_{k}^{(n-1)}-\cdots-A_{n} Z_{k}^{(1)} .
\end{aligned}\right.
$$

Para simplificar a notação, vamos escrever

$$
Z_{k}=\left[\begin{array}{c}
Z_{k}^{(1)} \\
\vdots \\
Z_{k}^{(n)}
\end{array}\right], \tilde{B}_{k}=\left[\begin{array}{c}
0 \\
\vdots \\
0 \\
B_{k}
\end{array}\right] \text { e } \mathcal{C}=\left[\begin{array}{ccccc}
0 & I & 0 & \cdots & 0 \\
0 & 0 & I & \cdots & 0 \\
\vdots & \vdots & \vdots & \ddots & \vdots \\
0 & 0 & 0 & \cdots & I \\
-A_{n} & -A_{n-1} & -A_{n-2} & \cdots & -A_{1}
\end{array}\right]
$$

onde $Z_{k}$ e $\tilde{B}_{k}$ são vetores-colunas por blocos em $\mathbb{C}^{m n \times m}$ e $\mathcal{C}$ é uma matriz por blocos ${ }^{1}$ em $\mathbb{C}^{m n \times m n}$.

\footnotetext{
${ }^{1} \mathrm{~A}$ matriz $\mathcal{C}$ é chamada matriz companheira por blocos associada à equação (1.5).
} 
O sistema anterior pode ser escrito como

$$
Z_{k+1}=\mathcal{C} Z_{k}+\tilde{B}_{k}, \quad k=0,1, \ldots,
$$

que é uma equação de diferença matricial linear de ordem 1 , cuja solução pode ser obtida recursivamente e é dada por

$$
Z_{k}=\mathcal{C}^{k} Z_{0}+\sum_{j=0}^{k-1} \mathcal{C}^{k-1-j} \tilde{B}_{j}, \quad \text { onde } k=0,1, \ldots
$$

Em particular, se $\left\{B_{k}\right\}$ é uma seqüência constante com $B_{k}=B$, e se $(I-\mathcal{C})$ é não-singular, temos

$$
Z_{k}=\mathcal{C}^{k} Z_{0}+(I-\mathcal{C})^{-1}\left(I-\mathcal{C}^{k}\right) \hat{B}
$$

onde $\tilde{B}=[0, \ldots, 0, B]^{t}$.

De uma forma geral, a solução de (1.5) é dada por

$$
Y_{k}=[I, 0, \ldots, 0] Z_{k} .
$$

A soluçâo descrita em (1.7) é chamada solução geral, pois envolve uma matriz constante arbitrária, $Z_{0}$. Se determinarmos essa matriz constante através de condições iniciais, obtemos uma solução particular de (1.6).

A reduçào da equação (1.5) a um sistema linear de $1 \underline{a}$ ordem não simplifica o problema de determinar sua solução numericamente, pois a expressão (1.7) envolve o cálculo de potências da matriz por blocos $\mathcal{C}$.

Vamos descrever um método para determinar as potências de uma matriz através do Teorema de Cayley-Hamilton. Seja $p_{A}(\lambda)=\operatorname{det}(\lambda I-A)$ o polinômio característico de $A \in \mathbb{C}^{n \times n}$ e seja $k \geq 0$ fixado. Da divisão de $\lambda^{k}$ por $p_{A}(\lambda)$, obtemos

$$
\lambda^{k}=p_{A}(\lambda) q(\lambda)+r(\lambda)
$$


onde $q(\lambda)$ e $r(\lambda)$ são os polinômios quociente e resto, respectivamente, da divisão de $\lambda^{k}$ por $p_{A}(\lambda)$. Observamos que o grau $r(\lambda)$ é no máximo $n-1$, logo,

$$
r(\lambda)=r_{0}+r_{1} \lambda+\cdots+r_{n-1} \lambda^{n-1} .
$$

Se $\lambda=A$, pelo Teorema de Cayley-Hamilton, $p_{A}(A)=0$, ou seja,

$$
A^{k}=r(A)=r_{0}+r_{1} A+\cdots+r_{n-1} A^{n-1} \text {. }
$$

Os coeficientes $r_{i}$ podem ser determinados como se segue :

i) Se $A$ tem todos os valores próprios distintos, $\lambda_{1}, \ldots, \lambda_{n}$, então em (1.8),

$$
\lambda_{i}^{k}=r\left(\lambda_{i}\right), \quad i=1, \ldots, n,
$$

que é um sistema de equações lineares em $\lambda_{i}$, dado por

$$
\left\{\begin{aligned}
\lambda_{1}^{k} & =r_{0}+r_{1} \lambda_{1}+\cdots+r_{n-1} \lambda_{1}^{n-1} \\
& \vdots \\
\lambda_{n}^{k} & =r_{0}+r_{1} \lambda_{n}+\cdots+r_{n-1} \lambda_{n}^{n-1},
\end{aligned}\right.
$$

ou seja,

$$
\left(\begin{array}{cccc}
1 & \lambda_{1} & \ldots & \lambda_{1}^{n-1} \\
1 & \lambda_{2} & \ldots & \lambda_{2}^{n-1} \\
\vdots & \vdots & \ddots & \vdots \\
1 & \lambda_{n} & \ldots & \lambda_{n}^{n-1}
\end{array}\right)\left(\begin{array}{c}
r_{0} \\
r_{1} \\
\vdots \\
r_{n-1}
\end{array}\right)=\left(\begin{array}{c}
\lambda_{1}^{k} \\
\lambda_{2}^{k} \\
\vdots \\
\lambda_{n}^{k}
\end{array}\right)
$$

O sistema acima admite uma única solução $r_{0}, \ldots, r_{n-1}$, pois a matriz dos coeficientes é não-singular (é a matriz de Vandermonde transposta - ver Seção 2.3).

ii) Suponhamos que nem todos os valores próprios de $A$ são distintos, por exemplo, $\lambda_{1}, \ldots, \lambda_{q}$ são valores próprios distintos de $A$ de multiplicidades $\nu_{1}, \ldots, \nu_{q}$, respectivamente, tal que $\sum_{i=1}^{q} \nu_{i}=n$. Nesse caso, o sistema de equações obtido em (i) é indeterminado. A fim de determinar as constantes $r_{i}$ univocamente, vamos considerar o sistema de equações composto de $q$ sub-sistemas da forma : 


$$
\left\{\begin{array}{c}
r\left(\lambda_{i}\right)=\lambda_{i}^{k} \\
\vdots \\
r^{\left(\nu_{i}-1\right)}\left(\lambda_{i}\right)=k(k-1) \cdots\left(k-\nu_{i}+2\right) \lambda_{i}^{k-\nu_{i}+1},
\end{array}\right.
$$

onde $i=1, \ldots, q$ e $r^{(j)}\left(\lambda_{i}\right)=\left.\frac{d^{j}}{d \lambda^{j}} r(\lambda)\right|_{\lambda=\lambda_{i}}$. Para simplificar a notação, vamos escrever $g(\lambda)=\lambda^{k}$. O sub-sistema acima fica :

$$
\left\{\begin{array}{rrrr}
r_{0}+r_{1} \lambda_{i}+\cdots+ & r_{\nu_{i}-1} \lambda_{i}^{\nu_{i}-1}+\cdots+ & r_{n-1} \lambda_{i}^{n-1}= & g\left(\lambda_{i}\right) \\
r_{1}+\cdots+ & \left(\nu_{i}-1\right) r_{\nu_{i}-1} \lambda_{i}^{\nu_{i}-2}+\cdots+ & (n-1) r_{n-1} \lambda_{i}^{n-2}= & g^{\prime}\left(\lambda_{i}\right) \\
& \vdots & \\
\left(\nu_{i}-1\right) ! r_{\nu_{i}-1}+\cdots+ & \left(\begin{array}{c}
n-1 \\
n-\nu_{i}+1
\end{array}\right) r_{n-1} \lambda_{i}^{n-\nu_{i}}= & g^{\left(\nu_{i}-1\right)}\left(\lambda_{i}\right) .
\end{array}\right.
$$

Matricialmente, escrevemos

$$
\begin{gathered}
M\left(\lambda_{i}\right)=\left(\begin{array}{cccccc}
1 & \lambda_{i} & \ldots & \lambda_{i}^{\nu_{i}-1} & \ldots & \lambda_{i}^{n-1} \\
0 & 1 & \ldots & \left(\nu_{i}-1\right) \lambda_{i}^{\nu_{i}-2} & \ldots & (n-1) \lambda_{i}^{n-2} \\
\vdots & \vdots & & \vdots & & \vdots \\
0 & 0 & \ldots & \left(\nu_{i}-1\right) ! & \ldots & \left(\begin{array}{c}
n-1 \\
\nu_{i}-1
\end{array}\right) \lambda_{i}^{n-\nu_{i}}
\end{array}\right) \in \mathbb{C}^{\nu_{i} \times n} \\
\text { e } G\left(\lambda_{i}\right)=\left(\begin{array}{c}
g\left(\lambda_{i}\right) \\
g^{\prime}\left(\lambda_{i}\right) \\
\vdots \\
g^{\left(\nu_{i}-1\right)}\left(\lambda_{i}\right)
\end{array}\right) \in \mathbb{C}^{\nu_{i} \times 1} .
\end{gathered}
$$

O sistema completo, considerando-se todos os valores próprios $\lambda_{1}, \ldots, \lambda_{q}$, pode ser escrito como :

$$
\begin{array}{r}
M\left(r_{0}, \cdots, r_{n-1}\right)^{t}=G \\
\text { onde } M=\left[\begin{array}{c}
M\left(\lambda_{1}\right) \\
\vdots \\
M\left(\lambda_{q}\right)
\end{array}\right] \in \mathbb{C}^{n \times n} \text { e } G=\left[\begin{array}{c}
G\left(\lambda_{1}\right) \\
\vdots \\
G\left(\lambda_{q}\right)
\end{array}\right] \in \mathbb{C}^{n \times 1} .
\end{array}
$$


A matriz $M$ é a matriz de Vandermonde generalizada transposta e o sistema (1.10) admite uma única solução $r_{0}, \ldots, r_{n-1}$, já que os valores próprios $\lambda_{1}, \ldots, \lambda_{q}$ são distintos (ver Seção 2.3).

Logo, podemos determinar $A^{j}, j=0, \ldots, n-1$ através da equação (1.9) e assim calcular $A^{k}$, que é uma combinação linear de $I, A, \ldots, A^{n-1}$ com os coeficientes $r_{0}, \ldots, r_{n-1}$ obtidos em (i) ou (ii).

Do ponto de vista prático, este processo só faz sentido se as dimensões do problema forem pequenas, uma vez que envolve o cálculo de todos os valores próprios da matriz $\mathcal{C}$.

Para caracterizar o conjunto das soluçòes de uma equaçào de diferença matricial linear homogênea $\mathcal{M}_{H}$, vamos introduzir a definiçào da matriz de Cassorati e seu determinante, chamado cassoratiano, que é equivalente ao wronskiano das equações diferenciais. No que se segue, usaremos a seguinte notaçào : $\operatorname{col}\left(X_{i}\right)_{i=m}^{p}=\left[\begin{array}{c}X_{m} \\ \vdots \\ X_{m+p}\end{array}\right]$.

Definição 1.5 : Sejam $\left\{Y_{k}^{(1)}\right\}, \ldots,\left\{Y_{k}^{(n)}\right\} \in \mathcal{M}$. Para cada $k$ fixado, consideremos a matriz

$$
\Phi_{k}=\left[\operatorname{col}\left(Y_{j}^{(1)}\right)_{j=k}^{n-1}, \operatorname{col}\left(Y_{j}^{(2)}\right)_{j=k}^{n-1}, \ldots, \operatorname{col}\left(Y_{j}^{(n)}\right)_{j=k}^{n-1},\right] \in \mathbb{C}^{m n \times m n},
$$

onde col $\left(Y_{k}^{(j)}\right)_{j=k}^{n-1}$ é um vetor-coluna por blocos em $\mathbb{C}^{m n \times m}$, para $j=1, \ldots, n$. Chamamos $\Phi_{k}$ de matriz de Cassorati e o determinante de $\Phi_{k}$ é chamado de cassoratiano associado às matrizes $Y_{k}^{(i)}, i=1, \ldots, n$. Denotaremos $c_{k}=\operatorname{det} \Phi_{k}$.

Observamos que $\left\{c_{k}\right\}$ é uma seqüência numérica. 
Definição 1.6 : Sejam $\left\{Y_{k}^{(1)}\right\}, \ldots,\left\{Y_{k}^{(n)}\right\}$ soluções da equação

$$
Y_{k+n}+A_{1} Y_{k+n-1}+\cdots+A_{n} Y_{k}=0, \quad k=0,1, \ldots
$$

Se $\left\{c_{k}\right\}$, a seqüência de cassoratianos associada a essas soluções, é não-nula, então o conjunto $\left\{\left\{Y_{k}^{(1)}\right\}, \ldots,\left\{Y_{k}^{(n)}\right\}\right\}$ é chamado um conjunto fundamental de soluçôes de (1.11).

Esta definição coincide com a dada usualmente para conjunto fundamental de soluções de uma equação de diferença linear no caso escalar. Vamos manter a mesma. denominação para os dois conjuntos sem risco de confusão, mas lembrando que, no caso escalar, o conjunto fundamental de soluções é uma base do subespaço das soluções de uma equação de diferença e, como veremos adiante, o mesmo não ocorre no caso matricial.

A proposiçào seguinte mostra uma propriedade interessante que relaciona o cassoratiano a uma equação de diferença linear de ordem 1.

Proposição 1.1: Se $\psi=\left\{\left\{Y_{k}^{(1)}\right\}, \ldots,\left\{Y_{k}^{(n)}\right\}\right\}$ é um conjunto fundamental de soluçôes de (1.11) e $\left\{c_{k}\right\}$ é a seqüência de cassoratianos associada a $\psi$, então

$$
c_{k+1}=(\operatorname{det} \mathcal{C}) c_{k}, \quad \text { para } k=0,1, \ldots,
$$

onde $\mathcal{C}$ é a matriz companheira por blocos associada à equaçâo de diferença (1.11).

Demonstração : Seja $\Phi_{k}$ como na Definição 1.5. Observe que $\mathcal{C} \Phi_{k}=\Phi_{k+1}$, logo, $\operatorname{det} \Phi_{k+1}=(\operatorname{det} \mathcal{C})\left(\operatorname{det} \Phi_{k}\right)$. Como $\operatorname{det} \Phi_{k}=c_{k}$, temos que $c_{k+1}=(\operatorname{det} \mathcal{C}) c_{k}$.

Como $\operatorname{det} \mathcal{C}=(-1)^{n} \operatorname{det} A_{n}$, também podemos escrever que

$$
c_{k+1}=(-1)^{n}\left(\operatorname{det} A_{n}\right) c_{k} .
$$


Observação 1.2 : Considere o seguinte problema de Cauchy :

$$
\left\{\begin{array}{l}
c_{k+1}=(\operatorname{det} \mathcal{C}) c_{k}, \\
c_{s}=a, \text { para algum } s \geq 0 .
\end{array}\right.
$$

Se $c_{s}=0$, pelo Corolário 1.1 do Teorema de Existência e Unicidade, segue-se que $c_{k}=0$ para $k=0,1, \ldots$. Assim sendo, para que $\left\{c_{k}\right\}$ seja a seqüencia nula, basta que $c_{s}=0$ para algum $s \geq 0$.

Finalizando este capítulo, a proposição abaixo mostra como gerar uma solução da equação (1.11) a partir de um conjunto fundamental de soluções.

Proposição 1.2: Seja $\psi=\left\{\left\{Y_{k}^{(1)}\right\}, \ldots,\left\{Y_{k}^{(n)}\right\}\right\}$ um conjunto fundamental de soluçôes de (1.11). Entâo $\sum_{i=1}^{n} Y_{k}^{(i)} T_{i}$ é solução de (1.11) e toda soluçâo de (1.11) é da forma $\sum_{i=1}^{n} Y_{k}^{(i)} T_{i}, k=0,1, \ldots$, onde $T_{i} \in \mathbb{C}^{m \times m}$ são constantes.

Demonstraçâo: Verifica-se por substituição que a seqüência $\left\{Y_{k}\right\} \in \mathcal{M} \operatorname{com} Y_{k}=$ $\sum_{i=1}^{n} Y_{k}^{(i)} T_{i}$ é uma solução de (1.11). Por outro lado, se $\left\{X_{k}\right\}$ é uma solução não-nula de (1.11) e se $\left\{c_{k}\right\}$ é a seqüência de cassoratianos associada a $\psi$, vamos mostrar que $X_{k}$ é da forma $\sum_{i=1}^{n} Y_{k}^{(i)} T_{i}$.

Como $\psi$ é um conjunto fundamental de soluções, existe $k_{0}$ tal que $c_{k_{0}} \neq 0$, logo o sistema de equaçôes matriciais

$$
\left\{\begin{array}{c}
Y_{k_{0}}^{(1)} T_{1}+\cdots+Y_{k_{0}}^{(n)} T_{n}=X_{k_{0}} \\
\vdots \\
Y_{k_{0}+n-1}^{(1)} T_{1}+\cdots+Y_{k_{0}+n-1}^{(n)} T_{n}=X_{k_{0}+n-1}
\end{array}\right.
$$

admite uma soluçào $T_{i}, i=1, \ldots, n$, não trivial. Seja $Z_{k}=\sum_{i=1}^{n} Y_{k}^{(i)} T_{i}$ uma solução de (1.11). Em particular, se $k=k_{0}$,

$$
\begin{aligned}
Z_{k_{0}} & =X_{k_{0}} \\
& \vdots \\
Z_{k_{0}+n-1} & =X_{k_{0}+n-1},
\end{aligned}
$$


logo, pelo Teorema de Existência e Unicidade, $X_{k}=Z_{k}, k=0,1 \ldots$, ou seja,

$$
X_{k}=\sum_{i=1}^{n} Y_{k}^{(i)} T_{i}, \quad k=0,1, \ldots .
$$

Neste ponto, aparecem as primeiras diferenças entre a teoria das equações de diferença lineares escalares e matriciais, a começar pelas soluções. No caso escalar, o conjunto das soluções forma um subespaço vetorial complexo e uma base é dada. por um conjunto fundamental de soluçôes. Já no caso matricial, o conjunto de soluções $\mathcal{M}_{H}$ tem estrutura de subespaço vetorial, mas não podemos considerar um conjunto fundamental de soluções como uma base. Isso porque $\sum_{i=1}^{n} Y_{k}^{(i)} T_{i}$ não é uma combinação linear de $Y_{k}^{-(i)}, \quad i=1, \ldots, n$, no sentido usualmente conhecido, já que as $T_{i}$ 's sào matrizes constantes em $\mathbb{C}^{m \times m}$ e o conjunto das matrizes quadradas não é um corpo. Para ser mais específico, podemos dizer que $\mathcal{M}_{H}$ é um módulo. Como $\mathbb{C}^{m \times m}$ é um anel com unidade e $\mathcal{M}_{H}$ é um grupo aditivo comutativo, o conjunto $\mathcal{M}_{H}$ é um $\mathbb{C}^{m \times m}$-módulo à direita. E, como foi mostrado na Proposição 1.2, as constantes $T_{i}$ que aparecem na soluçào geral $\sum_{i=1}^{n} Y_{k}^{(i)} T_{i}$ não podem ser escalares em $\mathbb{C}$ a não ser em casos muito particulares.

Observação 1.3 : A solução geral da equação de diferença à esquerda

$$
Y_{k+n}+Y_{k+n-1} A_{1}+\cdots+Y_{k} A_{n}=0
$$

é da forma $\sum_{i=1}^{n} T_{i} Y_{k}^{(i)}$, onde $Y_{k}^{(i)}$ pertence a um conjunto fundamental dessa equação, desde que tal conjunto exista. 


\section{Capítulo 2}

\section{LAMBDA-MATRIZ E POLINÔMIO MATRICIAL}

Neste capítulo, apresentamos algumas definiçôes e resultados sobre a teoria dos polinômios matriciais e lambda-matrizes que serão utilizados nos capítulos seguintes. Maiores detalhes podem ser encontrados em [2], [4] e [12]. Também apresentamos uma generalização das matrizes de Vandermonde [16].

\subsection{Lambda-matriz}

Um método que desenvolveremos no Capítulo 5 para obtenção de uma solução de uma equação de diferença linear consiste na aplicação da transformada de Laurent a essa equação, transformando-a em uma equação algébrica, à semelhança do que faz a transformada de Laplace em uma equação diferencial. Após determinar a solução dessa equação algébrica, é necessário o uso da transformada inversa de Laurent, e uma forma fechada para a solução da equação de diferença será obtida com o uso das lambda-matrizes. Nesta seção, apresentamos um breve resumo sobre as lambda-matrizes. 
Consideremos uma matriz com $m$ linhas e $r$ colunas cujos elementos são polinômios na variável $\lambda$, onde $\lambda$ é um escalar complexo. Essa matriz também pode ser escrita como um polinômio de coeficientes matriciais, chamado de lambda-matriz ou $\lambda$-matriz.

Definição 2.1 : Sejam $A_{0}, \ldots, A_{n}$ matrizes em $\mathbb{C}^{m \times r}$. O polinômio

$$
p(\lambda I)=p(\lambda)=A_{0} \lambda^{n}+A_{1} \lambda^{n-1}+\cdots+A_{n} \in \mathbb{C}^{m \times r}
$$

é chamado de $\lambda$-matriz. Denotamos cada elemento de $p(\lambda)$ por $p_{i j}(\lambda)$. Se $A_{0}$ é matriz não-nula, dizemos que o grau de $p(\lambda)$ é $n$.

Definição 2.2 : Se $r=m$ e $A_{0}$ é não-singular, entào $p(\lambda)$ é uma $\lambda$-matriz regular.

As operaçòes de adição e multiplicação entre $\lambda$-matrizes são as mesmas definidas para matrizes em geral. O passo seguinte é analisar a inversibilidade de $p(\lambda)$. Daqui em diante, consideramos somente as $\lambda$-matrizes quadradas de dimensão $m$. Da teoria dos determinantes, temos a seguinte identidade :

$$
p(\lambda)(\operatorname{adj} p(\lambda))=(\operatorname{det} p(\lambda)) I=(\operatorname{adj} p(\lambda)) p(\lambda),
$$

onde det $p(\lambda)$ é o determinante de $p(\lambda)$ e adj $p(\lambda)$ é uma $\lambda$-matriz de grau $n(m-1)$ cujos elementos são os cofatores de $p(\lambda)$.

Se $p(\lambda)$ é não-singular, então $p(\lambda)$ é inversivel e

$$
p^{-1}(\lambda)=\frac{\operatorname{adj} p(\lambda)}{\operatorname{det} p(\lambda)} \text {. }
$$

O problema de determinar os valores de $\lambda$ para os quais uma $\lambda$-matriz é inversível nos leva às seguintes definiçòes.

Definição 2.3 : Seja $p(\lambda)$ uma $\lambda$-matriz. Chamamos raiz latente de $p(\lambda)$ ao escalar $\lambda \in \mathbb{C}$ tal que det $p(\lambda)=0$. 
Definição 2.4 : Seja $\lambda_{0}$ uma raiz latente de $p(\lambda)$. Chamamos de vetor latente $\grave{a}$ direita de $p(\lambda)$ associado a $\lambda_{0}$ a todo vetor $\mathrm{u}=\left(u_{1}, \ldots, u_{m}\right)^{t} \in \mathbb{C}^{m \times 1}$ que é solução não-trivial do sistema de equações :

$$
p\left(\lambda_{0}\right) \mathrm{u}=(0, \ldots, 0)^{t} .
$$

De modo análogo, chamamos o vetor $\mathrm{v}=\left(v_{1}, \ldots, v_{m}\right) \in \mathbb{C}^{1 \times m}$ de vetor latente $\grave{a}$ esquerda de $p(\lambda)$ associado a $\lambda_{0}$ se $\mathrm{v}$ é solução não-trivial do sistema de equações :

$$
\mathrm{v} p\left(\lambda_{0}\right)=(0, \ldots, 0)
$$

Observamos que para uma matriz $A$ constante, as raizes latentes de $(A-\lambda I)$ sào os valores próprios de $A$, valendo relaçào análoga para os vetores latentes e os vetores próprios.

Vejamos como definir a divisão entre $\lambda$-matrizes (ver [3]). Sejam as $\lambda$-matrizes em $\mathbb{C}^{m \times m}$ dadas por $p(\lambda)=A_{0} \lambda^{n}+A_{1} \lambda^{n-1}+\cdots+A_{n}$ e $d(\lambda)=B_{0} \lambda^{t}+B_{1} \lambda^{t-1}+\cdots+B_{t}$ onde $d(\lambda)$ é regular e $t \leq n$. Então existem e são únicas as $\lambda$-matrizes $q_{1}(\lambda)$ e $r_{1}(\lambda)$ tais que

$$
p(\lambda)=q_{1}(\lambda) d(\lambda)+r_{1}(\lambda),
$$

onde o grau de $r_{1}$ é menor que $n$. A expressão acima define a divisão à direita de $p(\lambda)$ por $d(\lambda)$, onde $q_{1}(\lambda)$ é o quociente à direita de $p(\lambda)$ e $r_{1}(\lambda)$ é o resto à direita de $p(\lambda)$ na divisão por $d(\lambda)$.

Também podemos definir divisão à esquerda de $p(\lambda)$ por $d(\lambda)$ :

$$
p(\lambda)=d(\lambda) q_{2}(\lambda)+r_{2}(\lambda)
$$

onde $q_{2}(\lambda)$ é o quociente à esquerda e $r_{2}(\lambda)$ é o resto à esquerda.

O processo de divisào à esquerda e à direita é único, desde que $d(\lambda)$ seja regular. 


\subsection{Polinômio matricial}

O método clássico para obtenção de soluções de uma equação de diferença linear no caso vetorial ( ver Seção 3.1) consiste em associar uma equação de diferença a um polinômio, e através de suas raízes construir as soluções. Como nosso objetivo no capítulo seguinte é também associar um polinômio matricial a uma equação de diferença matricial linear, apresentamos nesta seção algumas definições e resultados sobre os polinômios matriciais. Maiores detalhes podem ser obtidos em [2] e [5].

Definição 2.5 : Sejam $A_{0}, \ldots, A_{n}, \mathcal{Z} \in \mathbb{C}^{m \times m}$. Entâo

$$
p(\mathcal{Z})=A_{0} \mathcal{Z}^{n}+A_{1} \mathcal{Z}^{n-1}+\cdots+A_{n}
$$

é chamado de polinômio matricial. Se $A_{0}$ é matriz não-nula, dizemos que o grau do polinômio acima é $n$.

Observamos que

$$
\hat{p}(\mathcal{Z})=\mathcal{Z}^{n} A_{0}+\mathcal{Z}^{n-1} A_{1}+\cdots+A_{n}
$$

também é um polinômio matricial, de ordem $n$ se $A_{0}$ é nâo-nula.

Definição 2.6 : Se $p(\mathcal{S})=0$, então $\mathcal{S}$ é um solvente à direita de $p(\mathcal{Z})$, e se $\hat{p}(\mathcal{R})=$ 0 , então $\mathcal{R}$ é um solvente à esquerda de $p(\mathcal{Z})$.

Notamos que as $\lambda$-matrizes são um caso particular dos polinômios matriciais, onde $\mathcal{Z}=\lambda I$. No caso de lambda-matrizes, podemos relacioná-las com os solventes através de um corolário do Teorema generalizado de Bezóut, cuja demonstração pode ser encontrada em [12].

Teorema 2.1 (Bezóut) Seja $p(\lambda)$ uma $\lambda$-matriz e seja $\mathcal{S} \in \mathbb{C}^{m \times m}$. Então

$$
p(\lambda)=q(\lambda)(\lambda I-\mathcal{S})+p(\mathcal{S})
$$

onde grau $q<$ grau $p$. 
Corolário 2.1 : Seja $p(\lambda)$ uma $\lambda$-matriz e seja $\mathcal{S} \in \mathbb{C}^{m \times m}$ um solvente à direita de $p(\mathcal{Z})$. Entâo

$$
p(\lambda)=q_{1}(\lambda)(\lambda I-\mathcal{S})
$$

onde $q_{1}(\lambda)$ é uma $\lambda$-matriz de grau $n-1$.

Observação 2.1 : Analogamente, se $\mathcal{R} \in \mathbb{C}^{m \times m}$, temos

$$
p(\lambda)=(\lambda I-\mathcal{R}) q_{2}(\lambda)+\hat{p}(\mathcal{R}),
$$

e no caso de $\mathcal{R}$ ser um solvente à esquerda de $p(\mathcal{Z})$, tem-se

$$
p(\lambda)=(\lambda I-\mathcal{R}) q_{2}(\lambda)
$$

onde $q_{2}(\lambda)$ é uma $\lambda$-matriz de grau $n-1$.

Devido às expressòes (2.1) e (2.2) é que chamamos $\mathcal{S}$ de solvente à clireita de $p(\mathcal{Z})$ e $\mathcal{R}$ de solvente à esquerda de $p(\mathcal{Z})$, respectivamente. A relação entre $\mathcal{S}$ e $\mathcal{R}$ pode ser encontrada em [2].

Pelo teorema a.cima,

$$
\operatorname{det} p(\lambda)=\left(\operatorname{det} q_{1}(\lambda)\right)(\operatorname{det}(\lambda I-\mathcal{S})),
$$

logo, o solvente $\mathcal{S}$ tem $n$ valores próprios que fazem parte das $m n$ raízes latentes de $p(\lambda)$. Agora, vamos responder à seguinte pergunta : de posse das raízes latentes de $p(\lambda)$, podemos obter um solvente? A resposta é dada pela proposição abaixo .

Proposição 2.1 ([12]) Seja $p(\lambda)$ uma $\lambda$-matriz de grau $n$ que tem $m$ vetores latentes linearmente independentes $\mathrm{u}_{1}, \ldots, \mathrm{u}_{m} \in \mathbb{C}^{m \times 1}$, correspondentes a $m$ raízes latentes $\lambda_{1}, \ldots, \lambda_{m} \in \mathbb{C}$, nào necessariamente distintos. Se $U=\left(\mathrm{u}_{1}, \ldots, \mathrm{u}_{m}\right) \in \mathbb{C}^{m \times m}$ e $\Lambda=\operatorname{diag}\left(\lambda_{1}, \ldots, \lambda_{m}\right) \in \mathbb{C}^{m \times m}$, então $U \Lambda U^{-1}$ é um solvente de $p(\mathcal{Z})$.

Demonstração: Seja $p(\lambda)=\sum_{i=0}^{n} A_{i} \lambda^{n-i}$. Como $p\left(\lambda_{i}\right) u_{i}=0$, temos que

$$
\begin{aligned}
A_{0} U \Lambda^{n}+A_{1} U \Lambda^{n-1}+\cdots+A_{n} U & =\sum_{j=1}^{m} A_{0} \mathbf{u}_{j} \lambda_{j}^{n}+\cdots+\sum_{j=1}^{m} A_{n} \mathbf{u}_{j} \\
& =\sum_{j=1}^{m} \sum_{i=0}^{n} A_{i} \mathbf{u}_{j} \lambda_{j}^{n-i}=0 .
\end{aligned}
$$


Multiplicando a equação acima por $U^{-1}$ :

$$
\left(A_{0} U \Lambda^{n}+A_{1} U \Lambda^{n-1}+\cdots+A_{n} U\right) U^{-1}=0
$$

e usando que $U \Lambda^{i} U^{-1}=\left(U \Lambda U^{-1}\right)^{i}$, segue que

$$
p\left(U \Lambda^{i} U^{-1}\right)=\sum_{i=0}^{n} A_{i}\left(U \Lambda U^{-1}\right)^{n-i}=0 .
$$

Corolário 2.2 : Seja $\lambda_{1}, \ldots, \lambda_{m n}$ o conjunto de mn raizes latentes distintas de $p(\lambda)$ tal que todo conjunto de $m$ vetores latentes associados é linearmente independente (l.i.), isto é, satisfaz a condiçấo de Haar. Então existem exatamente $\left(\begin{array}{c}m n \\ m\end{array}\right)$ solventes distintos de $p(\mathcal{Z})$.

Com este corolário, verificamos que um polinômio matricial nâo satisfaz o Teorema fundamental da Álgebra, pois pode admitir um número de solventes bem maior que o seu grau se o conjunto dos vetores latentes satisfaz a condição de Haar (considere por exemplo, a equação matricial $X^{2}=X$ ). Por outro lado, o problema. da raiz quadrada $X^{2}=A, A \in \mathbb{C}^{m \times m}$, mostra que um polinômio matricial pode não admitir nenhum solvente [4].

Agora, vamos definir multiplicidade de um solvente.

Definição 2.7 : Seja $\nu \in \mathbb{Z}_{+}, \nu \geq 1$. Dizemos que $\mathcal{S} \in \mathbb{C}^{m \times m}$ é um solvente de $p(\mathcal{Z})$ com multiplicidade $\nu$ se $p^{(j)}(\mathcal{S})=0$ para ${ }_{j=0, \ldots, \nu-1}$ e $p^{(\nu)}(\mathcal{S}) \neq 0$, onde convencionamos que $p^{(0)}(\lambda)=p(\lambda)$ e que $p^{(j)}(\lambda)=\frac{d^{\jmath}}{d \lambda^{\prime}} p(\lambda)$.

Observação 2.2 : Em nosso trabalho, consideraremos $A_{0}=I$ nas definiçòes de $\lambda$-matriz e polinômio matricial.

Em particular, se $p(\lambda)=\lambda^{n} I+\sum_{i=1}^{n} A_{i} \lambda^{n-i}$, temos

$$
p^{(i)}(\lambda)=\left\{\begin{array}{c}
j !\left[\left(\begin{array}{c}
n \\
j
\end{array}\right) \lambda^{n-j}+\sum_{i=1}^{n-j} A_{n-i}\left(\begin{array}{c}
n-i \\
j
\end{array}\right) \lambda^{n-i-j}\right], \text { se } j \leq n \\
0, \quad \text { se } j>n .
\end{array}\right.
$$




\subsection{Matriz de Vandermonde por blocos}

Para encerrar este capítulo, vamos falar sobre a matriz de Vandermonde e a matriz de Vandermonde generalizada. Estas matrizes surgem na resolução de certos sistemas de equaçòes lineares.

Definição 2.8 : Dados $z_{1}, \ldots, z_{n} \in \mathbb{C}$, a matriz

$$
v\left(z_{1}, \ldots, z_{n}\right)=\left(\begin{array}{ccc}
1 & \ldots & 1 \\
z_{1} & \ldots & z_{n} \\
\vdots & \ddots & \vdots \\
z_{1}^{n-1} & \ldots & z_{n}^{n-1}
\end{array}\right) \in \mathbb{C}^{n \times n}
$$

é chamada matriz de Vandermonde associada a $z_{1}, \ldots, z_{n}$.

No Capítulo 1, a matriz acima apareceu ao descrevermos um método para o cálculo de potências de matrizes. Observamos que se os escalares $z_{i}$ são distintos, então

$$
\operatorname{det} v\left(z_{1}, \ldots, z_{n}\right)=\prod_{1 \leq i<j \leq n}\left(z_{i}-z_{j}\right) \neq 0
$$

ou seja, $v\left(z_{1}, \ldots, z_{n}\right)$ é nào-singular.

Por outro lado, no mesmo exemplo vimos como pode surgir a matriz de Vandermonde generalizada.

Definição 2.9 : Seja $\tilde{p}(z)$ um polinômio de grau $n$ e sejam $z_{1}, \ldots, z_{q} \in \mathbb{C}$ raízes distintas de $\tilde{p}(z)$ de multiplicidades $\nu_{1}, \ldots, \nu_{q}$ onde $\sum_{i=1}^{q} \nu_{i}=n, \operatorname{com} \nu_{i} \geq 1$. Para cada $s=1, \ldots, q$, seja $w_{s}$ uma matriz em $\mathbb{C}^{m \times \nu_{s}}$ dada por

$$
w_{s}=\left(w_{i j}^{(s)}\right)=\left\{\begin{array}{cc}
\left(\begin{array}{c}
i-1 \\
i-j
\end{array}\right) z_{s}^{i-j} & , i \geq j \\
0 & , i<j
\end{array} .\right.
$$


A matriz $\left(w_{1}, \ldots, w_{q}\right) \in \mathbb{C}^{n \times n}$ é chamada matriz de Vandermonde generalizada associada $a z_{1}, \ldots, z_{q}$ e é representada por $v_{n}\left(z_{1}, \ldots, z_{q}\right)=\left(w_{1}, \ldots, w_{q}\right)$. O subíndice $n$ na notação $v_{n}$ indica o número de linhas dessa matriz.

Por exemplo, se $p(z)=(z-a)^{3}(z-b)^{2}$, então

$$
v_{5}(a, b)=\left(\begin{array}{ccccc}
1 & 0 & 0 & 1 & 0 \\
a & 1 & 0 & b & 1 \\
a^{2} & 2 a & 1 & b^{2} & 2 b \\
a^{3} & 3 a^{2} & 3 a & b^{3} & 3 b^{2} \\
a^{4} & 4 a^{3} & 6 a^{2} & b^{4} & 4 b^{3}
\end{array}\right) .
$$

Observamos que

$$
\operatorname{det} v_{n}\left(z_{1}, \ldots, z_{q}\right)=\prod_{1 \leq i<j \leq q}\left(z_{j}-z_{i}\right)^{m_{i} m_{\jmath}}
$$

e, se, em particular, todos os zeros de $p(z)$ são distintos, a matriz de Vandermonde generalizada se reduz à matriz de Vandermonde usual. Mais detalhes podem ser obtidos em [10].

Agora, vamos estender as definiçôes acima, pois vamos utilizá-la no Capítulo 3 para determinar em que condições obtemos um conjunto fundamental de soluçòes de uma equaçào de diferença matricial linear homogênea.

Definição 2.10 : Sejam $\mathcal{S}_{1}, \ldots, \mathcal{S}_{n} \in \mathbb{C}^{m \times m}$. A matriz de Vandermonde por blocos associada a $\mathcal{S}_{1}, \ldots, \mathcal{S}_{n}$ é uma matriz em $\mathbb{C}^{m n \times m n}$ denotada por $V\left(\mathcal{S}_{1}, \ldots, \mathcal{S}_{n}\right)$, e é dada por :

$$
V\left(\mathcal{S}_{1}, \ldots, \mathcal{S}_{n}\right)=\left[\begin{array}{ccc}
I & \ldots & I \\
\mathcal{S}_{1} & \ldots & \mathcal{S}_{n} \\
\vdots & \ddots & \vdots \\
\mathcal{S}_{1}^{n-1} & \ldots & \mathcal{S}_{n}^{n-1}
\end{array}\right]
$$


Não podemos afirmar que $\operatorname{det} V\left(\mathcal{S}_{1}, \ldots, \mathcal{S}_{n}\right) \neq 0$ mesmo que as $\mathcal{S}_{i}$ 's ou que os valores próprios das $\mathcal{S}_{i}$ 's sejam distintas, isso devido à não-comutatividade das matrizes. Considere o seguinte exemplo [2] :

$$
\begin{gathered}
\mathcal{S}_{1}=\left(\begin{array}{cc}
2 & 0 \\
-2 & 1
\end{array}\right), \mathcal{S}_{2}=\left(\begin{array}{ll}
4 & 2 \\
0 & 3
\end{array}\right) \\
\text { mas det } V\left(\mathcal{S}_{1}, \mathcal{S}_{2}\right)=\left|\begin{array}{cc}
I & I \\
\mathcal{S}_{1} & \mathcal{S}_{2}
\end{array}\right|=\left|\mathcal{S}_{2}-\mathcal{S}_{1}\right|=\left|\begin{array}{ll}
2 & 2 \\
2 & 2
\end{array}\right|=0 .
\end{gathered}
$$

Em [8], Jódar apresenta critérios para a não-singularidade da matriz de Vandermonde por blocos.

Suponhamos que as matrizes $\mathcal{S}_{1}, \ldots, \mathcal{S}_{q}$ são solventes de $p(\mathcal{Z})=\mathcal{Z}^{n}+A_{1} \mathcal{Z}^{n-1}+$ $\cdots+A_{n}$ com multiplicidade maior ou igual a 1 . Vamos definir a matriz de Vandermonde generalizada por blocos associado a esse conjunto, estendendo a definição (2.9).

Definição 2.11 : Sejam $\mathcal{S}_{1}, \ldots, \mathcal{S}_{q}$ solventes de $p(\mathcal{Z})$ com multiplicidade $\nu_{1}, \ldots, \nu_{q}$, respectivamente, onde $1 \leq q \leq n$. Seja. $W_{i} \in \mathbb{C}^{m n \times \nu_{i} m}, \quad i=1, \ldots, q$, onde :

$$
W_{i}=\left[\begin{array}{cclc}
I & 0 & \ldots & 0 \\
\mathcal{S}_{i} & I & \ldots & 0 \\
\mathcal{S}_{i}^{2} & 2 \mathcal{S}_{i} & \ldots & 0 \\
\vdots & \vdots & & \vdots \\
\mathcal{S}_{i}^{\nu_{i}-2} & \left(\nu_{i}-2\right) \mathcal{S}_{i}^{\nu_{i}-3} & \ldots & 0 \\
\mathcal{S}_{i}^{\nu_{i}-1} & \left(\nu_{i}-1\right) \mathcal{S}_{i}^{\nu_{i}-2} & \ldots & \left(\nu_{i}-1\right) ! I \\
\vdots & \vdots & & \vdots \\
\mathcal{S}_{i}^{n-1} & (n-1) \mathcal{S}_{i}^{n-2} & \ldots & \left(\nu_{i}-1\right) !\left(\begin{array}{c}
n-1 \\
n-\nu_{i}
\end{array}\right) \mathcal{S}_{i}^{n-\nu_{i}}
\end{array}\right]
$$

Definimos a matriz de Vandermonde generalizada por blocos $V_{n}\left(\mathcal{S}_{1}, \ldots, \mathcal{S}_{q}\right)$ por

$$
\left[W_{1}, \ldots, W_{q}\right] \in \mathbb{C}^{m n \times m\left(\nu_{1}+\cdots+\nu_{q}\right)},
$$


onde o sub-índice $n$ em $V_{n}\left(\mathcal{S}_{1}, \ldots, \mathcal{S}_{q}\right)$ se refere ao número de blocos existentes em cada linha.

Em [16], Maroulas apresenta critérios para a não-singularidade de $V_{n}$. 


\section{Capítulo 3}

\section{CONJUNTO FUNDAMENTAL DE SOLUÇÕES}

Neste capítulo, determinamos em que condiçôes podemos encontrar um conjunto fundamental de soluçôes para uma equação de diferença matricial linear tomando por base a teoria das equaçòes de diferença linear vetorial.

Na Seção 3.1, apresentamos um método para obtenção de soluções de uma equação de diferença linear homogênea (caso vetorial), chamado método clássico. $\mathrm{Na}$ Seção 3.2, mostramos como devem ser os elementos de um conjunto fundamental de soluções.

\subsection{Método clássico}

Seja a equação

$$
\mathrm{y}_{k+n}+A_{1} \mathrm{y}_{k+n-1}+\cdots+A_{n} \mathrm{y}_{k}=0
$$

onde $A_{i} \in \mathbb{C}^{m \times m}$ e $\mathrm{y}_{k} \in \mathbb{C}^{m \times 1}$. Uma solução de (3.1) é uma seqüência de vetores $\left\{\mathrm{y}_{k}\right\}, \operatorname{com} \mathrm{y}_{k} \in \mathbb{C}^{m \times 1}$, que satisfaz (3.1).

Considere o problema de valores iniciais :

Problema de Cauchy: Dadas as condições iniciais $\mathrm{c}_{0}, \ldots, \mathrm{c}_{n-1} \in \mathbb{C}^{m \times 1}$, determinar uma soluçào de (3.1) tal que $\mathrm{y}_{0}=\mathrm{c}_{0}, \ldots, \mathrm{y}_{n-1}=\mathrm{c}_{n-1}$. 
O Teorema de Existência e Unicidade demonstrado no Capítulo 1 também se aplica ao problema acima, fazendo as devidas adaptaçôes nas dimensões das matrizes. Pode-se mostrar [1\$] que a solução geral de (3.1) é da forma

$$
\mathrm{y}_{k}=a_{1} \mathrm{y}_{k}^{(1)}+\cdots+a_{n} \mathbf{y}_{k}^{(n)}
$$

onde $a_{i} \in \mathbb{C}$ e $\left\{\mathrm{y}_{k}^{(j)}\right\}_{k=0}^{\infty}, j=1, \ldots n$ são soluções linearmente independentes (l.i.) de (3.1).

As soluções $\left\{\mathrm{y}_{k}^{(j)}\right\}_{k=0}^{\infty}$ podem ser obtidas através do método clássico, que consiste em determinar o valor de $\alpha \in \mathbb{C}, \alpha \neq 0$ tal que $\alpha^{k}$ c é uma solução de (3.1), onde $\mathrm{c} \in \mathbb{C}^{m \times 1}$ é um vetor constante arbitrário.

Se $\mathrm{y}_{k}=a^{k} \mathrm{c}$ é uma solução de (3.1), então

$$
\left(\alpha^{n} I+\alpha^{n-1} A_{1}+\cdots+A_{n}\right) \alpha^{k} \mathrm{c}=0 .
$$

Como $\alpha \neq 0$ e c é uma constante arbitrária, devemos ter

$$
\alpha^{n} I+a^{n-1} A_{1}+\cdots+A_{n}=0 .
$$

Esta equação é chamada de equação característica de (3.1). Logo, se $\alpha$ é uma raiz simples de (3.2), a seqüência $\left\{\alpha^{k} \mathbf{c}\right\}$ é uma solução de (3.1). Para obtermos todas as soluçòes l.i. de (3.1), devemos analisar a multiplicidade das raízes de (3.2). Se $\alpha$ tem multiplicidade $\nu \geq 1$, além de $\left\{\alpha^{k} \mathbf{c}\right\}$, temos como soluçòes as seqüências $\left\{\mathbf{y}_{k}\right\}$ com $\mathbf{y}_{k}=k^{j} \alpha^{k} \mathbf{c}$ ou $\mathbf{y}_{k}=\left(\begin{array}{l}k \\ j\end{array}\right) \alpha^{k-i} \mathbf{c}$, para $j=0, \ldots, \nu-1$. Dessa forma, um conjunto fundamental de soluçòes de (3.1) consiste em $n$ seqüências dadas por $\left\{\mathbf{y}_{k}\right\}$ descritas acima.

\subsection{Conjunto fundamental de soluções}

Iniciamos esta seção com a definição de polinômio característico associado a uma equaçào de diferença matricial linear homogênea. Vamos mostrar que as soluções que formam um conjunto fundamental de soluçòes podem ser construídas de forma. análoga ao caso vetorial, apresentado acima. 
Definição 3.1 : Seja a equação

$$
Y_{k+n}+A_{1} Y_{k+n-1}+\cdots+A_{n} Y_{k}=0, \quad k=0,1, \ldots
$$

Chamamos de polinômio característico de (3.3) ao polinômio matricial

$$
p(\mathcal{Z})=\mathcal{Z}^{n}+A_{1} \mathcal{Z}^{n-1}+\cdots+A_{n}
$$

onde $\mathcal{Z}, A_{1}, \ldots, A_{n} \in \mathbb{C}^{m \times m}$.

Observação 3.1 : Associamos a matriz companheira por blocos $\mathcal{C}$ ao polinômio matricial $p(\mathcal{Z})$, pois det $(\mathcal{C}-\lambda I)=(-1)^{m n} \operatorname{det} p(\mathcal{Z})$ é chamado de polinômio característico.

Como vimos na Seçâo 2.2, um polinômio matricial não se comporta como um polinômio escalar de ordem $n$, que admite sempre $n$ raízes. Pelo contrário, um polinômio matricial pode tanto nào admitir nenhum solvente como admitir bem mais que $n$ solventes. A fim de entendermos o significado, neste caso, de conjunto fundamental de soluçòes, vejamos primeiro o que acontece com uma equação de diferença matricial linear homogênea de ordem 2 para, em seguida, generalizarmos este conceito para as equaçòes de diferença de ordem $n$.

Seja a equaçào

$$
Y_{k+2}+A_{1} Y_{k+1}+A_{2} Y_{k}=0 \quad k=0,1, \ldots,
$$

onde $A_{1}, A_{2} \in \mathbb{C}^{m \times m}$ e seja $p(\mathcal{Z})=\mathcal{Z}^{2}+A_{1} \mathcal{Z}+A_{2}$ o polinômio característico associado.

a) Se $\mathcal{S}_{1}$ e $\mathcal{S}_{2}$ sào solventes de $p(\mathcal{Z})=\mathcal{Z}^{2} I+A_{1} \mathcal{Z}+A_{2}$ tais que $\left(\mathcal{S}_{2}-\mathcal{S}_{1}\right)$ é não-singular, então $\left\{\mathcal{S}_{1}^{k}\right\}$ e $\left\{\mathcal{S}_{2}^{k}\right\}$ formam um conjunto fundamental de soluçōes de (3.4).

b) Se $\mathcal{S}_{1}$ é um solvente de $p(\mathcal{Z})$ tal que $2 \mathcal{S}_{1}+A_{1}=0$, então $\left\{\mathcal{S}_{1}^{k}\right\}$ e $\left\{k \mathcal{S}_{1}^{k-1}\right\}$ formam um conjunto fundamental de soluções de (3.4).

Verificamos (a) e (b) da seguinte forma : 
a) As seqüências $\left\{\mathcal{S}_{1}^{k}\right\}$ e $\left\{\mathcal{S}_{2}^{k}\right\}$ sâo soluções de (3.4). Pela Definiçào 1.5, o cassoratiano associado a essas soluções em $k=0$ é dado por

$$
c_{0}=\left|\begin{array}{cc}
I & I \\
\mathcal{S}_{1} & \mathcal{S}_{2}
\end{array}\right| .
$$

Mas $c_{0}=\operatorname{det}\left(\mathcal{S}_{2}-\mathcal{S}_{1}\right) \neq 0$ por hipótese, logo, pela observação $1.2, c_{k} \neq 0$ para todo $k$ e portanto, $\left\{\mathcal{S}_{1}^{k}\right\}$ e $\left\{\mathcal{S}_{2}^{k}\right\}$ formam um conjunto fundamental de soluções .

b) $\left\{Y_{k}\right\}=\left\{k \mathcal{S}_{1}^{k-1}\right\}$ é soluçào de (3.4), pois :

$$
\begin{aligned}
Y_{k+2}+A_{1} Y_{k+1}+A_{2} Y_{k} & =(k+2) \mathcal{S}_{1}^{k+1}+A_{1}(k+1) \mathcal{S}_{1}^{k}+A_{2} k \mathcal{S}_{1}^{k-1} \\
& =\left[k\left(\mathcal{S}_{1}^{2}+A_{1} \mathcal{S}_{1}+A_{2}\right)+\left(2 \mathcal{S}_{1}+A_{1}\right) \mathcal{S}_{1}\right] \mathcal{S}_{1}^{k-1} \\
& =\left[k p\left(\mathcal{S}_{1}\right)+\left(2 \mathcal{S}_{1}+A_{1}\right) \mathcal{S}_{1}\right] \mathcal{S}_{1}^{k-1} \\
& =0, \quad k=0,1, \ldots
\end{aligned}
$$

Agora, o cassoratiano associado às soluçôes $\left\{\mathcal{S}_{1}^{k}\right\}$ e $\left\{k \mathcal{S}_{1}^{k-1}\right\}$ para $k$ fixado é dado por

$$
c_{k}=\left|\begin{array}{cc}
\mathcal{S}_{1}^{k} & k \mathcal{S}_{1}^{k-1} \\
\mathcal{S}_{1}^{k+1} & (k+1) \mathcal{S}_{1}^{k}
\end{array}\right| .
$$

Em $k=0$ temos $c_{0}=\left|\begin{array}{cc}I & 0 \\ \mathcal{S}_{1} & I\end{array}\right| \neq 0$, donde segue que $\left\{\left\{\mathcal{S}_{1}^{k}\right\},\left\{k \mathcal{S}_{1}^{k-1}\right\}\right\}$ é um conjunto fundamental de soluçòes.

A condiçào $2 \mathcal{S}_{1}+A_{1}=0$ caracteriza um solvente de multiplicidade dois. De fato, usando que $A_{1}=-2 \mathcal{S}_{1}$ em $p\left(\mathcal{S}_{1}\right)=0$, obtemos $A_{2}=\mathcal{S}_{1}^{2}$, e assim

$$
p(\lambda)=\lambda^{2} I+A_{1} \lambda+A_{2}=\lambda^{2} I-2 \mathcal{S}_{1} z+\mathcal{S}_{1}^{2}=\left(\lambda I-\mathcal{S}_{1}\right)^{2}
$$

A generalizaçâo para a equação de diferença matricial linear homogênea de ordem $n$ pode ser feita como se segue. Seja $p(\mathcal{Z})=\mathcal{Z}^{n}+A_{1} \mathcal{Z}^{n-1}+\cdots+A_{n}$ o polinômio 
característico associado à equação de diferença matricial linear de ordem $n$ :

$$
Y_{k+n}+A_{1} Y_{k+n-1}+\cdots+A_{n} Y_{k}=0, \quad k=0,1, \ldots .
$$

Suponhamos que $p(\mathcal{Z})$ admita $n$ solventes distintos. Nesse caso, temos a seguinte generalização do resultado anterior :

Teorema 3.1: Sejam $\mathcal{S}_{1}, \ldots, \mathcal{S}_{n} \in \mathbb{C}^{m \times m}$ solventes distintos de $p(\mathcal{Z})$. Se a matriz de Vandermonde por blocos associada a $\mathcal{S}_{1}, \ldots, \mathcal{S}_{n}, V\left(\mathcal{S}_{1}, \ldots, \mathcal{S}_{n}\right)$, é não-singular, então $\left\{\left\{Y_{k}^{(1)}\right\}, \ldots,\left\{Y_{k}^{(n)}\right\}\right\}$, onde $Y_{k}^{(i)}=\mathcal{S}_{i}^{k}, i=1, \ldots, n$, é um conjunto fundamental de soluçôes de (3.5).

Demonstraçào: Através de substituição, verificamos que para cada $i=1, \ldots, n$, a seqüência $\left\{\mathcal{S}_{i}^{k}\right\}_{k=0}^{\infty}$ é solução de (3.5). Vamos construir o cassoratiano associado às soluções $\left\{Y_{k}^{(i)}\right\}$. Seja $k$ fixado. Pela Definição 1.5 ,

$$
\begin{gathered}
c_{k}=\operatorname{det}\left[\operatorname{col}\left(\mathcal{S}_{1}^{j}\right)_{j=k}^{n-1}, \operatorname{col}\left(\mathcal{S}_{2}^{j}\right)_{j=k}^{n-1}, \ldots, \operatorname{col}\left(\mathcal{S}_{n}^{j}\right)_{j=k}^{n-1}\right], \\
\text { ou seja, } c_{k}=\operatorname{det}\left[\begin{array}{ccc}
\mathcal{S}_{1}^{k} & \ldots & \mathcal{S}_{n}^{k} \\
\mathcal{S}_{1}^{k+1} & \ldots & \mathcal{S}_{n}^{k+1} \\
\vdots & \ddots & \vdots \\
\mathcal{S}_{1}^{k+n-1} & \ldots & \mathcal{S}_{n}^{k+n-1}
\end{array}\right]
\end{gathered}
$$

Como por hipótese $c_{0}=\operatorname{det} V\left(\mathcal{S}_{1}, \ldots, \mathcal{S}_{n}\right) \neq 0$, segue que $\left\{\left\{\mathcal{S}_{i}^{k}\right\}, \quad i=1, \ldots, n\right\}$ é um conjunto fundamental de soluçòes.

Vamos considerar agora o caso em que os solventes têm multiplicidade maior ou igual a 1. Primeiro, vejamos como construir soluçôes de uma equação de diferença linear a partir de solventes múltiplos. Para cada solvente $\mathcal{S} \in \mathbb{C}^{m \times m}$ de multiplicidade $\nu \geq 1$ construímos um conjunto $\operatorname{com} \nu$ seqüências da forma

$$
\left\{\left\{\mathcal{S}^{k}\right\},\left\{k \mathcal{S}^{k-1}\right\}, \ldots,\left\{\left(\begin{array}{c}
k \\
\nu-1
\end{array}\right) \mathcal{S}^{k-\nu+1}\right\}\right\} .
$$

A proposiçào seguinte mostra que cada uma das seqüências acima é solução da. equaçào (3.5). 
Proposição 3.1 : Seja $p(\mathcal{Z})$ o polinômio característico associado a (3.5) e seja $\mathcal{S} \in \mathbb{C}^{m \times m}$ um solvente de $p(\mathcal{Z})$ com multiplicidade $\nu$. Então as seqüências $\left\{Y_{k}^{(0)}\right\}, \ldots,\left\{Y_{k}^{(\nu-1)}\right\}$ tais que

$$
Y_{k}^{(j)}=\left\{\begin{array}{cc}
j !\left(\begin{array}{c}
k \\
j
\end{array}\right) \mathcal{S}^{k-j} & , k \geq j \\
0 & , k<j
\end{array}\right.
$$

são soluçòes de (3.5) para $j=0, \ldots, \nu-1$.

Demonstraçâo: Seja $0 \leq j \leq \nu-1$. Devemos mostrar que $Y_{k}^{(j)}=j !\left(\begin{array}{l}k \\ j\end{array}\right) \mathcal{S}^{k-j}$ satisfaz a equaçào (3.5), ou seja,

$$
\begin{aligned}
Y_{k+n}^{(j)}+A_{1} Y_{k+n-1}^{(j)}+\cdots+A_{n} Y_{k}^{(j)}= & j !\left[\left(\begin{array}{c}
k+n \\
j
\end{array}\right) \mathcal{S}^{k+n-j}\right. \\
& \left.+\sum_{l=1}^{n} A_{l}\left(\begin{array}{c}
k+n-l \\
j
\end{array}\right) \mathcal{S}^{k+n-l-j}\right]=0 .
\end{aligned}
$$

Pela regra de Leibnitz para derivação, verificamos que

$$
\frac{d^{j}}{d \lambda^{j}}\left[p(\lambda) \lambda^{k}\right]=\sum_{i=0}^{j}(j-i) !\left(\begin{array}{l}
j \\
i
\end{array}\right) p^{(i)}(\lambda) \lambda^{k-j+i} .
$$

Por outro lado,

$$
\begin{aligned}
\frac{d^{3}}{d \lambda^{j}}\left[p(\lambda) \lambda^{k}\right] & =\frac{d^{3}}{d \lambda^{j}}\left[\lambda^{k+n} I+\sum_{l=1}^{n} A_{l} \lambda^{k+n-l}\right] \\
& =j !\left[\left(\begin{array}{c}
k+n \\
j
\end{array}\right) \lambda^{k+n-j} I+\sum_{l=1}^{n-j} A_{l}\left(\begin{array}{c}
k+n-l \\
j
\end{array}\right) \lambda^{k+n-l-j}\right] .
\end{aligned}
$$

Lembrando que $p(\mathcal{S})=\cdots=p^{(j)}(\mathcal{S})=0$, para $0 \leq j \leq \nu-1$, temos que se $\lambda=\mathcal{S}$,

$$
\begin{aligned}
0 & =\sum_{i=0}^{j}(j-i) !\left(\begin{array}{l}
j \\
i
\end{array}\right) p^{(i)}(\mathcal{S}) \mathcal{S}^{k-j+i} \\
& =\frac{d^{j}}{d \lambda^{j}}\left[p(\lambda) \lambda^{k}\right]_{\lambda=\mathcal{S}} \\
& =j !\left[\left(\begin{array}{c}
k+n \\
j
\end{array}\right) \mathcal{S}^{k+n-j}+\sum_{l=1}^{n-j} A_{l}\left(\begin{array}{c}
k+n-l \\
j
\end{array}\right) \mathcal{S}^{k+n-l-j}\right],
\end{aligned}
$$

que é exatamente a equaçào (3.6). 
O teorema seguinte mostra como determinar um conjunto fundamental de soluções a partir de solventes múltiplos.

Teorema 3.2 : Seja $p(\mathcal{Z})$ o polinômio característico associado a (3.5) e sejam $\mathcal{S}_{1}, \ldots, \mathcal{S}_{q}$ solventes de $p(\mathcal{Z})$ com multiplicidades $\nu_{1}, \ldots, \nu_{q}$, respectivamente, com $\nu_{i} \geq 1$, tal que $\sum_{i=1}^{q} \nu_{i}=n$. Se a matriz de Vandermonde por blocos generalizada $V_{n}\left(\mathcal{S}_{1}, \ldots, \mathcal{S}_{q}\right)$, é não-singular, então o conjunto

$$
\Psi=\left\{\left\{Y_{k}^{(0,1)}\right\}, \ldots,\left\{Y_{k}^{\left(\nu_{1}-1,1\right)}\right\}, \ldots \ldots,\left\{Y_{k}^{(0, q)}\right\}, \ldots,\left\{Y_{k}^{\left(\nu_{q}-1, q\right)}\right\}\right\}
$$

é um conjunto fundamental de soluções de (3.5), onde

$$
Y_{k}^{(j, i)}= \begin{cases}j !\left(\begin{array}{l}
k \\
j
\end{array}\right) \mathcal{S}_{i}^{k-j}, & k \geq j \\
0 & , k<j,\end{cases}
$$

com $i=1, \ldots, q$ e $0 \leq j \leq \nu_{i}-1$.

Demonstraçào: Já vimos anteriormente que as seqüências de $\Psi$ sào soluções de (3.5). Seja agora a matriz por blocos

$$
\Phi_{k}=\left[U_{k}^{(1)}, \ldots, U_{k}^{(q)}\right] \in \mathbb{C}^{m n \times m n},
$$

onde cada $U_{k}^{(i)} \in \mathbb{C}^{m n \times m \nu_{i}}$ é dada por

$$
U_{k}^{(i)}=\left[\begin{array}{ccc}
Y_{k}^{(0, i)} & \ldots & Y_{k}^{\left(\nu_{i}-1, i\right)} \\
\vdots & \ddots & \vdots \\
Y_{k+n-1}^{(0, i)} & \ldots & Y_{k+n-1}^{\left(\nu_{i}-1, i\right)}
\end{array}\right]
$$

O cassoratiano associado às seqüências de $\Psi$ em $k=0$ (conforme a Definiçào 1.5) é dado por

$$
c_{0}=\operatorname{det} \Phi_{0}=\operatorname{det}\left[U_{0}^{(1)}, \ldots, U_{0}^{(q)}\right]
$$




$$
\text { onde } U_{0}^{(i)}=\left[\begin{array}{cclc}
I & 0 & \ldots & 0 \\
\mathcal{S}_{i} & I & \ldots & 0 \\
\mathcal{S}_{i}^{2} & 2 \mathcal{S}_{i} & \ldots & 0 \\
\vdots & \vdots & & \vdots \\
\mathcal{S}_{i}^{\nu_{i}-2} & \left(\nu_{i}-2\right) \mathcal{S}_{i}^{\nu_{i}-3} & \ldots & 0 \\
\mathcal{S}_{i}^{\nu_{i}-1} & \left(\nu_{i}-1\right) \mathcal{S}_{i}^{\nu_{i}-2} & \ldots & \left(\nu_{i}-1\right) ! I \\
\vdots & \vdots & & \vdots \\
\mathcal{S}_{i}^{n-1} & (n-1) \mathcal{S}_{i}^{n-2} & \ldots & \left(\nu_{i}-1\right) !\left(\begin{array}{c}
n-1 \\
\nu_{i}-1
\end{array}\right) \mathcal{S}_{i}^{n-\nu_{i}}
\end{array}\right]
$$

$\operatorname{Em} k=0, c_{0}=\operatorname{det}\left[U_{0}^{(1)}, \ldots, U_{0}^{(q)}\right]=\operatorname{det} V_{n}\left(\mathcal{S}_{1}, \ldots, \mathcal{S}_{q}\right)$, onde $V_{n}\left(\mathcal{S}_{1}, \ldots, \mathcal{S}_{q}\right)$ é a matriz de Vandermonde por blocos generalizada. Como $c_{0} \neq 0$ por hipótese, segue que $\Psi$ é um conjunto fundamental de soluçôes.

Para encerrar este capítulo, consideremos o seguinte problema inverso : dado um conjunto de $n$ seqüências de matrizes, determinar em que condições obtemos uma. equação de diferença que admita esse conjunto como um conjunto fundamental de soluções. A resposta é dada pelo seguinte teorema. [15] :

Teorema 3.3 : Seja $X=\left\{\left\{X_{k}^{(1)}\right\}, \ldots,\left\{X_{k}^{(n)}\right\}\right\}$ um conjunto de $n$ seqüências de matrizes, $\left\{X_{k}^{(i)}\right\} \in \mathcal{M}, i=1, \ldots, n$, tal que o cassoratiano associado a $X$ nầ se anula. Entâo existe uma equação de diferença matricial que admite $X$ como conjunto fundamental de soluçòes.

Demonstraçâo : Seja $Y_{k+n}+A_{1} Y_{k+n-1}+\cdots+A_{n} Y_{k}=0, k=0,1, \ldots$ uma equação de diferença, onde $A_{j} \in \mathbb{C}^{m \times m}, j=1, \ldots, n$ são os coeficientes matriciais a determinar.

Seja $k$ fixado. O sistema linear de $n$ equações a $n$ incógnitas matriciais dado por

$$
X_{k+n}^{(i)}+\sum_{j=1}^{n} A_{j} X_{k+n-j}^{(i)}=0, i=1, \ldots, n,
$$

pode ser escrito matricialmente : 


$$
\left[A_{n}, \ldots, A_{1}\right]\left[\begin{array}{ccc}
X_{k}^{(1)} & \ldots & X_{k}^{(n)} \\
\vdots & \ddots & \vdots \\
X_{k+n-1}^{(1)} & \ldots & X_{k+n-1}^{(n)}
\end{array}\right]=\left[-X_{k+n}^{(1)}, \ldots,-X_{k+n}^{(n)}\right]
$$

onde, por hipótese, a matriz dos coeficientes desse sistema, $\Phi_{k}$, é não-singular. Desta forma, os coeficientes de $A_{1}, \ldots, A_{n}$ são determinados univocamente :

$$
\left[A_{n}, \ldots, A_{1}\right]=\left[-X_{k+n}^{(1)}, \ldots,-X_{k+n}^{(n)}\right] \Phi_{k}^{-1}
$$




\section{Capítulo 4}

\section{CADEIA DE SOLVENTES}

No capítulo anterior, seguindo os moldes do caso escalar, a determinaçào de soluçôes depende da obtenção de solventes. Vejamos agora um outro método de obter soluçào de uma equação de diferença matricial linear que faz uso da noção de cadeia de solventes [7]. O principal resultado deste capítulo é o Teorema 4.1, no qual mostramos que a matriz companheira por blocos é semelhante a uma matriz bi-diagonal por blocos.

\subsection{Cadeia de Solventes}

Definição 4.1 : Seja $p(\mathcal{Z})=\mathcal{Z}^{n}+A_{1} \mathcal{Z}^{n-1}+\cdots+A_{n}$ um polinômio matricial. Um conjunto de matrizes $\mathcal{S}_{1}, \ldots, \mathcal{S}_{n} \in \mathbb{C}^{m \times m}$ forma uma cadeia de solventes de $p(\mathcal{Z})$ se $p_{i}\left(\mathcal{S}_{i}\right)=0, i=1, \ldots, n$, onde

$$
\begin{aligned}
p_{0}(\lambda) & =I \\
p_{n}(\lambda) & =p(\lambda) \\
\text { e } \quad p_{i}(\lambda) & =p_{i-1}(\lambda)\left(\lambda I-\mathcal{S}_{i}\right), i=n, \ldots, 1
\end{aligned}
$$

Desta forma, $p(\lambda)=\left(\lambda I-\mathcal{S}_{1}\right) \cdots\left(\lambda I-\mathcal{S}_{n}\right)$. Em geral, somente $\mathcal{S}_{n}$ é um solvente à direita de $p(\mathcal{Z})$ (ou somente $\mathcal{S}_{1}$ é um solvente à esquerda de $p(\mathcal{Z})$ ). 
Reciprocamente, se $p(\lambda)=\prod_{i=1}^{n}\left(\lambda I-\mathcal{S}_{i}\right)$, então $\mathcal{S}_{i}, i=1, \ldots, n$ é uma cadeia de solventes.

Uma condiçâo suficiente para a existência de uma cadeia de solventes é dada pela proposição seguinte, cuja demonstração encontra-se em [2].

Proposição 4.1: Se as raizes latentes de $p(\lambda)$ são distintas, então $P(\mathcal{Z})$ admite uma cadeia de solventes.

Os lemas seguintes serào utilizados na demonstraçào do Teorema 4.1.

Lema 4.1: Seja $\mathcal{S}$ um solvente de $p(\mathcal{Z})=\mathcal{Z}^{n}+A_{1} \mathcal{Z}^{n-1}+\cdots+A_{n}$ tal que $p(\lambda)=$ $p_{n-1}(\lambda)(\lambda I-\mathcal{S})$. Entâo

$$
p_{n-1}(\lambda)=\lambda^{n-1} I+\sum_{i=1}^{n-1} B_{i} \lambda^{n-i-1},
$$

$\operatorname{com} B_{i}=B_{i-1} \mathcal{S}+A_{i}, i=1, \ldots, n-1$ e $B_{0}=I$.

Demonstraçìo : Como $p_{n-1}(\lambda)$ é uma $\lambda$-matriz de grau $n-1$, obtemos os coeficientes $B_{i}$ fazendo uma comparação entre os coeficientes de $p(\lambda)$ e de $p_{n-1}(\lambda)(\lambda I-\mathcal{S})$.

Lema 4.2: Sejam $\mathcal{S} \in \mathbb{C}^{m \times m}, J(\mathcal{S}) \in \mathbb{C}^{m n \times m n}$ e $p(\mathcal{Z})=\mathcal{Z}^{n}+A_{1} \mathcal{Z}^{n-1}+\ldots+A_{n}$ tais que $p(\mathcal{S})=0$ e

$$
J(S)=\left[\begin{array}{ccccc}
I & 0 & 0 & \cdots & 0 \\
\mathcal{S} & I & 0 & \cdots & 0 \\
\vdots & \vdots & \vdots & \ddots & \vdots \\
\mathcal{S}^{n-y 2} & \mathcal{S}^{n-3} & \mathcal{S}^{n-4} & \cdots & 0 \\
\mathcal{S}^{n-1} & \mathcal{S}^{n-2} & \mathcal{S}^{n-3} & \cdots & I
\end{array}\right]
$$

Então, a matriz companheira por blocos $\mathcal{C}$ associada a $p(\mathcal{Z})$ satisfaz a condição 


$$
J(\mathcal{S})^{-1} \mathcal{C} J(\mathcal{S})=F=\left[\begin{array}{c|cccc}
\mathcal{S} & I & 0 & \cdots & 0 \\
\hline 0 & & & \\
\vdots & & \mathcal{C}_{n-1} & \\
0 & & &
\end{array}\right]
$$

onde $\mathcal{C}_{n-1} \in \mathbb{C}^{m(n-1) \times m(n-1)}$ é a matriz companheira por blocos associada ao polinômio $p_{n-1}(\mathcal{Z})$ e $p(\lambda)=p_{n-1}(\lambda)(\lambda I-\mathcal{S})$.

Demonstraçào: Pelo Lema 4.1, a matriz companheira associada a $p_{n-1}(\mathcal{Z})$ é dada por

$$
\mathcal{C}_{n-1}=\left[\begin{array}{ccccc}
0 & I & 0 & \cdots & 0 \\
0 & 0 & I & \cdots & 0 \\
\vdots & \vdots & \vdots & \ddots & \vdots \\
0 & 0 & 0 & \cdots & I \\
-B_{n-1} & -B_{n-2} & -B_{n-3} & \cdots & -B_{1}
\end{array}\right]
$$

Então,

$$
\mathcal{C}(J(\mathcal{S}))=\left[\begin{array}{cccccc}
\mathcal{S} & I & 0 & \ldots & 0 & 0 \\
\mathcal{S}^{2} & \mathcal{S} & I & \ldots & 0 & 0 \\
\vdots & \vdots & \vdots & \ddots & \vdots & \vdots \\
\mathcal{S}^{n-1} & \mathcal{S}^{n-2} & \mathcal{S}^{n-3} & \ldots & \mathcal{S} & I \\
-R_{n}(\mathcal{S}) & -R_{n-1}(\mathcal{S}) & -R_{n-2}(\mathcal{S}) & \ldots & -R_{2}(\mathcal{S}) & -R_{1}(\mathcal{S})
\end{array}\right]
$$

onde $\left\{\begin{aligned} R_{n}(\mathcal{S}) & =A_{1} \mathcal{S}^{n-1}+A_{2} \mathcal{S}^{n-2}+\cdots+A_{n} \\ R_{n-1}(\mathcal{S}) & =A_{1} \mathcal{S}^{n-2}+A_{2} \mathcal{S}^{n-3}+\cdots+A_{n-1} \\ & \vdots \\ R_{1}(\mathcal{S}) & =A_{1} .\end{aligned}\right.$ 
Como $\mathcal{S}$ é um solvente de $p(\mathcal{Z})$, as equaçôes acima podem ser reescritas da seguinte forma :

$$
\left\{\begin{aligned}
R_{n}(\mathcal{S}) & =-\mathcal{S}^{n} \\
R_{n-1}(\mathcal{S}) & =B_{n-1}-\mathcal{S}^{n-1} \\
& \vdots \\
R_{1}(\mathcal{S}) & =B_{1}-\mathcal{S}
\end{aligned}\right.
$$

Por outro lado,

$$
J(\mathcal{S}) F=\left[\begin{array}{cccccc}
\mathcal{S} & I & 0 & \ldots & 0 & 0 \\
\mathcal{S}^{2} & \mathcal{S} & I & \ldots & 0 & 0 \\
\vdots & \vdots & \vdots & \ddots & \vdots & \vdots \\
\mathcal{S}^{n} & \mathcal{S}^{n-1} & \mathcal{S}^{n-2} & \ldots & \mathcal{S}^{2} & \mathcal{S}
\end{array}\right]
$$

Pelo sistema (4.1), segue que a última linha das matrizes $(J(\mathcal{S})) F$ e $\mathcal{C}(J(\mathcal{S}))$ coincidem. Logo, $(J(\mathcal{S})) F=\mathcal{C}(J(S))$.

Apresentamos agora o principal resultado deste capítulo :

Teorema $4.1([7])$ Se $p(\mathcal{Z})=\mathcal{Z}^{n}+\sum_{i=1}^{n} A_{i} \mathcal{Z}^{n-i}$ admite uma cadeia de solventes $\mathcal{S}_{1}, \ldots, \mathcal{S}_{n}$, entầo a matriz companheira por blocos $\mathcal{C}$ associada a $p(\mathcal{Z})$ é semelhante à matriz bi-diagonal por blocos:

$$
F_{n}=\left[\begin{array}{cccc}
\mathcal{S}_{n} & I & & \\
& \mathcal{S}_{n-1} & \ddots & \\
& & \ddots & I \\
& & & \mathcal{S}_{1},
\end{array}\right]
$$


onde $F_{n} \in \mathbb{C}^{m n \times m n}$.

Demonstração: Vamos adotar a seguinte notação : $\mathcal{C}_{n}$ é a matriz companheira associada a.o polinômio $p_{n}(\mathcal{Z})=p(\mathcal{Z})$. A demonstração segue por indução sobre $n$.

Seja $n=2$. Se $\mathcal{S}_{1}, \mathcal{S}_{2}$ é uma cadeia de solventes de $p_{2}(\mathcal{Z})$, podemos escrever

$$
p_{2}(\lambda)=\left(\lambda I-\mathcal{S}_{1}\right)\left(\lambda I-\mathcal{S}_{2}\right)
$$

Como $\mathcal{S}_{2}$ é um solvente é um solvente à direita de $p_{2}(\lambda)$, pelo Lema 4.1 , segue que

$$
p_{2}(\lambda)=\left(\lambda I+B_{1}\right)\left(\lambda I-\mathcal{S}_{2}\right)
$$

e a matriz companheira por blocos associada a $\left(\mathcal{Z}+B_{1}\right)$ é dada por $\mathcal{C}_{1}=-B_{1}$ e portanto, pelo Lema 4.2 segue que

$$
\mathcal{C}_{2} \sim\left[\begin{array}{cc}
\mathcal{S}_{2} & I \\
0 & -B_{1}
\end{array}\right]=\left[\begin{array}{cc}
\mathcal{S}_{2} & I \\
0 & \mathcal{S}_{1}
\end{array}\right]
$$

Como hipótese de indução, supomos que $\mathcal{C}_{n-1}$ é semelhante à matriz

$$
\left[\begin{array}{cccc}
\mathcal{S}_{n-1} & I & & \\
& \mathcal{S}_{n-2} & \ddots & \\
& & \ddots & I \\
& & & \mathcal{S}_{1},
\end{array}\right]
$$

Seja $p_{n}(\lambda)=p(\lambda)=\left(\lambda I-\mathcal{S}_{1}\right) \cdots\left(\lambda I-\mathcal{S}_{n}\right)$, onde $\mathcal{S}_{1}, \ldots, \mathcal{S}_{n}$ é uma cadeia de solventes de $p_{n}(\mathcal{Z})$. Como $\mathcal{S}_{n}$ é um solvente de $p_{n}(\lambda)$, pelo Lema 4.1 segue que $p_{n}(\lambda)=p_{n-1}(\lambda)\left(\lambda I-\mathcal{S}_{n}\right)$, onde $p_{n-1}(\lambda)=\left(\lambda I-\mathcal{S}_{1}\right) \cdots\left(\lambda I-\mathcal{S}_{n-1}\right)$. Pelo Lema 4.2 , temos

$$
\mathcal{C}_{n} \sim F=\left[\begin{array}{c|cccc}
\mathcal{S}_{n} & I & 0 & \cdots & 0 \\
\hline 0 & & & \\
\vdots & & \mathcal{C}_{n-1} & \\
0 & & &
\end{array}\right]
$$

logo, pela hipótese de induçào, segue que 


$$
\mathcal{C}_{n} \sim\left[\begin{array}{cccc}
\mathcal{S}_{n} & I & & \\
& \mathcal{S}_{n-1} & \ddots & \\
& & \ddots & I \\
& & & \mathcal{S}_{1}
\end{array}\right] .
$$

Proposição 4.2: Seja $\mathcal{S}_{1}, \ldots, \mathcal{S}_{n}$ uma cadeia de solventes de $p(\mathcal{Z})$ e seja $T_{n} \in$ $\mathbb{C}^{m n \times m n}$ tal que $T_{n}^{-1} \mathcal{C} T_{n}=F_{n}$, com $F_{n}$ como no teorema anterior. Então, $T_{n}=$ $J_{n} J_{n-1} \ldots J_{2}$, ondt

$$
\begin{gathered}
J_{n}=J\left(\mathcal{S}_{n}\right)=\left[\begin{array}{cccc}
I & 0 & \ldots & 0 \\
\mathcal{S}_{n} & I & \ldots & 0 \\
\vdots & \vdots & \ddots & \vdots \\
\mathcal{S}_{n}^{n-1} & \mathcal{S}_{n}^{n-2} & \ldots & I
\end{array}\right], \\
J_{n-1}=\operatorname{diag}\left[I, J\left(\mathcal{S}_{n-1}\right)\right], \\
\vdots \\
\left.J_{2}=\operatorname{diag}[\underbrace{I, \ldots, I}_{n-2}, J \mathcal{S}_{2})\right] .
\end{gathered}
$$

Demonstraçâo : A demonstração segue por induçào sobre $n$. Pelo Lema 4.2 temos que $\mathcal{C}_{2}=J\left(\mathcal{S}_{2}\right) F_{2} J\left(\mathcal{S}_{2}\right)^{-1}$. Suponhamos que $\mathcal{C}_{n-1}=P_{n-1} F_{n-1}\left(P_{n-1}\right)^{-1}$, onde

$$
P_{n-1}=J\left(\mathcal{S}_{n-1}\right) \operatorname{diag}\left[I, J\left(\mathcal{S}_{n-2}\right)\right] \cdots \operatorname{diag}[\underbrace{I, \cdots, I}_{n-3}, J\left(\mathcal{S}_{2}\right)]
$$

Seja $\mathcal{S}_{1}, \ldots, \mathcal{S}_{n}$ uma cadeia de solventes de $p(\mathcal{Z})$. Do Teorema anterior e do Lema 4.2 , segue que 


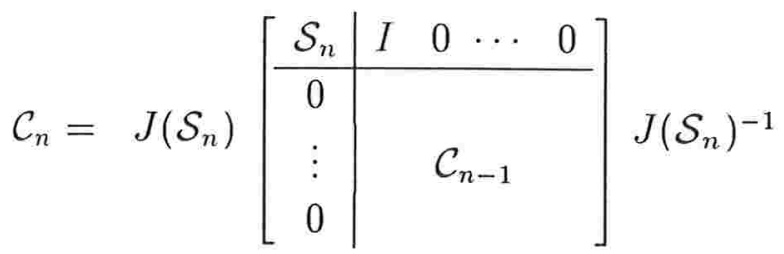

$$
\begin{aligned}
& =J\left(\mathcal{S}_{n}\right)\left[\begin{array}{c|cccc}
I & 0 & 0 & \cdots & 0 \\
\hline 0 & & & \\
\vdots & & P_{n-1} & \\
0 & & &
\end{array}\right]\left[\begin{array}{c|cccc}
\mathcal{S}_{n} & I & 0 & \cdots & 0 \\
\hline 0 & & & & \\
\vdots & & F_{n-1} & \\
0 & & &
\end{array}\right]\left[\begin{array}{c|cccc}
I & 0 & 0 & \cdots & 0 \\
\hline 0 & & & \\
\vdots & & P_{n-1}^{-1} & \\
0 & & &
\end{array}\right] J\left(\mathcal{S}_{n}\right)^{-1} \\
& =J\left(\mathcal{S}_{n}\right) \operatorname{diag}\left[I, P_{n-1}\right]\left[\begin{array}{c|cccc}
\mathcal{S}_{n} & I & 0 & \cdots & 0 \\
\hline 0 & & & \\
\vdots & & F_{n-1} & \\
0 & & &
\end{array}\right] \operatorname{diag}\left[I,\left(P_{n-1}\right)^{-1}\right] J\left(\mathcal{S}_{n}\right)^{-1} \\
& =T_{n} F_{n} T_{n}^{-1},
\end{aligned}
$$

$\operatorname{com} T_{n}=J\left(\mathcal{S}_{n}\right) J_{n-1} \cdots J_{2}$.

Observação 4.1: Os resultados abaixo serão utilizados a seguir. Por verificação direta, temos que:

$$
\begin{gathered}
T_{n}^{-1}=J_{2}^{-1} J_{3}^{-1} \ldots J_{n}^{-1}, \\
J_{k}^{-1}=\operatorname{diag}\left[I, \ldots, I, J\left(\mathcal{S}_{k}\right)^{-1}\right], \\
\text { onde } J\left(\mathcal{S}_{k}\right)^{-1}=\left[\begin{array}{ccccc}
I & & & & \\
-\mathcal{S}_{k} & I & & \\
& \ddots & \ddots & \\
& & -\mathcal{S}_{k} & I & \\
& & & -\mathcal{S}_{k} & I
\end{array}\right] .
\end{gathered}
$$


Vejamos como o Teorema 4.1 pode ser usado na obtenção de solução de um problema de valores iniciais para uma equaçâo de diferença matricial linear.

Teorema $4.2: S e j a$

$$
\left\{\begin{array}{l}
Y_{k+n}+A_{1} Y_{k+n-1}+\ldots+A_{n} Y_{k}=B_{k}, \quad k=0,1, \ldots \\
Y_{0}=D_{1}, \ldots, Y_{n-1}=D_{n}
\end{array}\right.
$$

um problema de valores iniciais, onde $D_{i} \in \mathbb{C}^{m \times m}$ são constantes. Seja $\mathcal{S}_{1}, \ldots, \mathcal{S}_{n}$ uma cadeia de solventes de $p(\mathcal{Z})=\mathcal{Z}_{n}+\sum_{i=1}^{n} A_{i} \mathcal{Z}_{n-i}$, então a solução de (4.2) é dada por :

$$
\begin{aligned}
Y_{k}=\mathcal{S}_{n}^{k} E_{1} & +\sum_{h_{1}=0}^{k-1} \mathcal{S}_{n}^{k-1-h_{1}} \mathcal{S}_{n-1}^{h_{1}} E_{2} \\
& +\sum_{h_{1}=0}^{k-1} \sum_{h_{2}=0}^{h_{1}-1} \mathcal{S}_{n}^{k-1-h_{1}} \mathcal{S}_{n-1}^{h_{1}-1-h_{2}} \mathcal{S}_{n-2}^{h_{2}} E_{3}+\ldots \\
& +\sum_{h_{1}=0}^{k-1} \sum_{h_{2}=0}^{h_{1}-1} \ldots \sum_{h_{n-1}=0}^{h_{n-2}-1} \mathcal{S}_{n}^{k-1-h_{1}} \mathcal{S}_{n-1}^{h_{1}-1-h_{2}} \ldots \mathcal{S}_{1}^{h_{n-1}} E_{n}+ \\
& +\sum_{h_{1}=0}^{k-1} \sum_{h_{2}=0}^{h_{1}-1} \ldots \sum_{h_{n}=0}^{h_{n-1}-1} \mathcal{S}_{n}^{k-1-h_{1}} \mathcal{S}_{n-1}^{h_{1}-1-h_{2}} \ldots \mathcal{S}_{1}^{h_{n-1}-1-h_{n}} B_{h_{n}},
\end{aligned}
$$

onde $\quad E_{i}=P_{i} T_{n}^{-1}\left[D_{1}, \ldots, D_{n}\right]^{t}$,

$P_{1}=[I, 0, \ldots, 0]$,

$P_{2}=[0,1, \ldots, 0]$,

$P_{n}=[0,0, \ldots, I]$

e $\quad T_{n}$ como na Proposição 4.2

Demonstraçào : Seja $k$ fixado. Fazendo a mudança de variáveis

$$
W_{k}^{(r)}=Y_{k+r-1}, \quad r=1, \ldots, n
$$


o problema (4.2) é equivalente a :

$$
\left\{\begin{aligned}
W_{k+1}^{(1)} & =W_{k}^{(2)} \\
W_{k+1}^{(2)} & =W_{k}^{(3)} \\
& \vdots \\
W_{k+1}^{(n)} & =Y_{k+1}=-A_{1} W_{k}^{(n)}-A_{2} W_{k}^{(n-1)}-\cdots-A_{n} W_{k}^{(1)}+B_{k} .
\end{aligned}\right.
$$

Simplificando a notação,

$$
\left\{\begin{aligned}
W_{k+1} & =\mathcal{C} W_{k}+\tilde{B}_{k} \\
W_{0} & =\left[D_{1}, \ldots, D_{n}\right]^{t}
\end{aligned}\right.
$$

onde $W_{k}=\left[\begin{array}{c}W_{k}^{(1)} \\ \vdots \\ W_{k}^{(n)}\end{array}\right], \tilde{B}_{k}=\left[\begin{array}{c}0 \\ \vdots \\ 0 \\ B_{k}\end{array}\right] \in \mathbb{C}^{m n \times m}$ e $\mathcal{C}$ é a matriz companheira por

blocos associada a $p(\mathcal{Z})$, polinômio característico de (4.2).

Como $p(\mathcal{Z})$ admite uma cadeia de solventes, pela Proposiçào 4.2 segue que $T_{n}^{-1} \mathcal{C} T_{n}=F_{n}$. Considerando a mudança de variáveis :

$$
W_{k}=T_{n} X_{k}
$$

em (4.4), obtemos $T_{n} X_{k+1}=\mathcal{C} T_{n} X_{k}+\dot{B}_{k}$, ou seja,

$$
\left\{\begin{aligned}
X_{k+1} & =T_{n}^{-1} \mathcal{C} T_{n} X_{k}+T_{n}^{-1} \tilde{B}_{k} \\
T_{n} X_{0} & =\left[D_{1}, \ldots, D_{n}\right]^{t}
\end{aligned}\right.
$$


Como $T_{n}^{-1} \tilde{B}_{k}=\tilde{B}_{k}$ e fazendo $T_{n}^{-1} X_{0}=\left[E_{1}, \ldots, E_{n}\right]^{t}$, o sistema (4.4) é equivalente a :

$$
\left\{\begin{array}{rl}
X_{k+1} & =F_{n} X_{k}+\tilde{B}_{k} \\
X_{0} & =\left[E_{1}, \ldots, E_{n}\right]^{t}
\end{array},\right.
$$

onde $X_{k}=\left[X_{k}^{(1)}, \ldots, X_{k}^{(n)}\right]^{t} \in \mathbb{C}^{m n \times m}$.

A solução de (4.2) é dada por :

$$
\begin{gathered}
Y_{k}=W_{k}^{(1)}=T_{n} X_{k}^{(1)}=X_{k}^{(1)} \\
{\left[\begin{array}{c}
X_{k+1}^{(1)} \\
\vdots \\
\vdots \\
\vdots \\
X_{k+1}^{(n)}
\end{array}\right]=\left[\begin{array}{lllll}
\mathcal{S}_{n} & I & & & \\
& \mathcal{S}_{n-1} & I & & \\
& & \ddots & \ddots & \\
& & & & I \\
& & & & Z_{1}
\end{array}\right]\left[\begin{array}{c}
X_{k}^{(1)} \\
\vdots \\
\vdots \\
\vdots \\
X_{k}^{(n)}
\end{array}\right]+\left[\begin{array}{c}
0 \\
\vdots \\
\vdots \\
0 \\
B_{k}
\end{array}\right]}
\end{gathered}
$$

Em termos de equaçòes, temos :

$$
\left\{\begin{array}{cc}
X_{k+1}^{(1)} & =\mathcal{S}_{n} X_{k}^{(1)}+X_{k}^{(2)} \\
X_{k+1}^{(2)} & =\mathcal{S}_{n-1} X_{k}^{(2)}+X_{k}^{(3)} \\
\vdots & \vdots \\
X_{k+1}^{(n-1)} & =\mathcal{S}_{2} X_{k}^{(n-1)}+X_{k}^{(n)} \\
X_{k+1}^{(n)} & =\mathcal{S}_{1} X_{k}^{(n)}+B_{k}
\end{array}\right.
$$

Vamos provar, por induçào sobre o número de equaçòes de (4.5), que a soluçâo é dada por (4.3). Considere as equaçōes (1) e (2) do sistema acima : 


$$
\left\{\begin{aligned}
X_{k+1}^{(n-1)} & =\mathcal{S}_{2} X_{k}^{(n-1)}+X_{k}^{(n)} \\
X_{k+1}^{(n)} & =\mathcal{S}_{1} X_{k}^{(n)}+B_{k}
\end{aligned}\right.
$$

A solução da equação (1), obtida recursivamente, é dada por :

$$
X_{k}^{(n)}=\mathcal{S}_{1}^{k} E_{n}+\sum_{h_{n}=0}^{k-1} \mathcal{S}_{1}^{k-1-h_{n}} B_{h_{n}} .
$$

Da mesma forma, a solução da equação (2) é :

$$
\begin{aligned}
X_{k}^{(n-1)}= & \mathcal{S}_{2}^{k} E_{n-1}+\sum_{h_{n-1}=0}^{k-1} \mathcal{S}_{2}^{k-1-h_{n-1}} X_{h_{n-1}}^{(n)} \\
= & \mathcal{S}_{2}^{k} E_{n-1}+\sum_{h_{n-1}=0}^{k-1} \mathcal{S}_{2}^{k-1-h_{n-1}}\left[\mathcal{S}_{1}^{h_{n-1}} E_{n}+\sum_{h_{n}=0}^{h_{n-1}-1} \mathcal{S}_{1}^{h_{n-1}-1-h_{n}} B_{h_{n}}\right] \\
= & \mathcal{S}_{2}^{k} E_{n-1}+\sum_{h_{n-1}=0}^{k-1} \mathcal{S}_{2}^{k-1-h_{n-1}} \mathcal{S}_{1}^{h_{n-1}} E_{n}+ \\
& \quad+\sum_{h_{n-1}=0}^{k-1} \sum_{h_{n}=0}^{h_{n-1}-1} \mathcal{S}_{2}^{k-1-h_{n-1}} \mathcal{S}_{1}^{h_{n-1}-1-h_{n}} B_{h_{n} .}
\end{aligned}
$$

Como hipótese de indução, supomos que a solução do seguinte sistema.

$$
\left\{\begin{aligned}
X_{k+1}^{(2)} & =\mathcal{S}_{n-1} X_{k}^{(2)}+X_{k}^{(3)} \\
\vdots & \vdots \\
X_{k+1}^{(n-1)} & =\mathcal{S}_{2} X_{k}^{(n-1)}+X_{k}^{(n)} \\
X_{k+1}^{(n)} & =\mathcal{S}_{1} X_{k}^{(n)}+B_{k}
\end{aligned}\right.
$$

tem por soluçào : 


$$
\begin{aligned}
X_{k}^{(2)}=\mathcal{S}_{n-1}^{k} E_{2} & +\sum_{h_{2}=0}^{k-1} \mathcal{S}_{n-1}^{k-1-h_{2}} \mathcal{S}_{n-2}^{h_{2}} E_{3} \\
& +\sum_{h_{2}=0}^{k-1} \sum_{h_{3}=0}^{h_{2}-1} \ldots \sum_{h_{n-1}=0}^{h_{n-2}-1} \mathcal{S}_{n-1}^{k-1-h_{2}} \mathcal{S}_{n-2}^{h_{2}-1-h_{3}} \ldots \mathcal{S}_{1}^{h_{n-2}} E_{n-1}+\ldots \\
& +\sum_{h_{2}=0}^{k-1} \sum_{h_{3}=0}^{h_{2}-1} \ldots \sum_{h_{n}=0}^{h_{n-1}-1} \mathcal{S}_{n-1}^{k-1-h_{2}} \ldots \mathcal{S}_{1}^{h_{n-1}-1-h_{n}} B_{h_{n}-1}
\end{aligned}
$$

Conseqüentemente, o sistema (4.5) admite como soluçâo :

$$
X_{k}^{(1)}=\mathcal{S}_{n}^{k} E_{1}+\sum_{h_{1}=0}^{k-1} \mathcal{S}_{n}^{k-1-h_{1}} X_{h_{1}}^{(2)} .
$$

Pela hipótese de induçào, segue que :

$$
\begin{aligned}
X_{k}^{(1)}=\mathcal{S}_{n}^{k} E_{1} & +\sum_{h_{1}=0}^{k-1} \mathcal{S}_{n}^{k-1-h_{1}} \mathcal{S}_{n-1}^{h_{1}} E_{2} \\
& +\sum_{h_{1}=0}^{k-1} \sum_{h_{2}=0}^{k-1} \mathcal{S}_{n}^{k-1-h_{1}} \mathcal{S}_{n-1}^{k-1-h_{2}} \mathcal{S}_{n-2}^{h_{2}} E_{3}+\ldots \\
& +\sum_{h_{1}=0}^{k-1} \sum_{h_{2}=0}^{h_{1}-1} \ldots \sum_{h_{n-1}=0}^{h_{n-2}-1} \mathcal{S}_{n}^{k-1-h_{1}} \mathcal{S}_{n-1}^{h_{1}-1-h_{2}} \ldots \mathcal{S}_{1}^{h_{n-1}} E_{n}+\ldots \\
& +\sum_{h_{1}=0}^{k-1} \sum_{h_{2}=0}^{h_{1}-1} \ldots \sum_{h_{n}=0}^{h_{n-1}-1} \mathcal{S}_{n}^{k-1-h_{1}} \mathcal{S}_{n-1}^{h_{1}-1-h_{2}} \ldots \mathcal{S}_{1}^{h_{n-1}-1-h_{n}} B_{h_{n}}
\end{aligned}
$$

o que conclui a demonstração, pois $Y_{k}=X_{k}^{(1)}, k=0,1, \ldots$. 


\section{Capítulo 5}

\section{TRANSFORMADA DE LAURENT MATRICIAL}

Neste capítulo, vamos desenvolver um outro método de soluçào das equações de diferença matriciais lineares. A transformada de Laurent ou $z$-transformada, como também é conhecida, tem muitas semelhanças com a transformada de Laplace tanto na definição como nas propriedades. Estamos interessados em utilizá-la para resolver equaçòes de diferenças, da mesma forma que a transformada de Laplace é utilizada. para resolver equaçöes diferenciais.

Apresentamos um breve resumo sobre a transformada de Laurent e a seguir, faremos a extensào desta, para aplicaçào em seqüências de matrizes. Mais detalhes sobre a transformada no caso escalar podem ser obtidos em [9], [13] e [19]. Também faremos uso de vários fatos da Análise Complexa [1].

\subsection{Transformada de Laurent - Caso Escalar}

Seja $\sigma=\left\{\left\{f_{k}\right\}_{k=0}^{\infty}, f_{k} \in \mathbb{C}^{\prime}\right\}$ o conjunto das seqüências numéricas. Por convenção, consideramos $f_{k}=0$ para $k<0$. A transformada de Laurent é uma. transformaçào definida sobre $\sigma$ que associa a cada elemento $\left\{f_{k}\right\} \in \sigma$ uma função 
$f(z), \operatorname{com} z \in \mathbb{C}$. Escrevemos

$$
l\left[\left\{f_{k}\right\}\right]=f(z) .
$$

Definição 5.1 : Seja $\left\{f_{k}\right\} \in \sigma$ uma seqüência numérica. A transformada de Laurent de $\left\{f_{k}\right\}$ é a funçào complexa dada por :

$$
l\left[\left\{f_{k}\right\}\right]=\sum_{k=0}^{\infty} f_{k} z^{-k}
$$

onde $z \in \mathbb{C}$ sâo valores para os quais a série acima converge.

Observamos que $\sum_{k=1}^{\infty} f_{k} z^{-k}$ é chamada de parte principal da série de Laurent dada por $\sum_{k=-\infty}^{\infty} f_{k} z^{-k}$, daí o nome da transformada. Também podemos construir uma transforma.da semelhante, considerando as potências positivas em $z$ da série da Laurent. Esta é conhecida como função geradora, e é dada por:

$$
\operatorname{ger}\left[\left\{f_{k}\right\}\right]=\sum_{k=0}^{\infty} f_{k} z^{k}=g(z) \text {. }
$$

A transformada de Laurent e a funçào geradora satisfazem a seguinte relaçào:

$$
f(z)=g\left(\frac{1}{z}\right) \text { ou } g(z)=f\left(\frac{1}{z}\right) .
$$

Salientamos que a preferência pela primeira deve-se ao fato de que a decomposiçào em fraçòes parciais, que será utilizada na inversa da transformada de Laurent, é mais natural nesse contexto ${ }^{1}$.

Definição 5.2 : Dizemos que $\left\{f_{k}\right\} \in \sigma$ é laurent-transformável se a série $\sum_{k=0}^{\infty} f_{k} z^{-k}$ é convergente, para algum $z \in \mathbb{C}$.

\footnotetext{
${ }^{1} \mathrm{Em}$ geral, a transformada de Laurent ou $z$-transformada é dada por $\sum_{k=-\infty}^{\infty} f_{k} z^{k}$. No nosso trabalho, esta se reduz à "one-sided transform", como definida em 5.1 , já que $f_{k}=0$ para $k<0$.
} 
A regiâo de convergência da série $\sum_{k=0}^{\infty} f_{k} z^{-k}$ é dada por

$$
\Omega=\{z \in \mathbb{C} /|z|>R\},
$$

onde $\frac{1}{R}=\varlimsup_{k \rightarrow \infty} \sqrt[k]{\left|f_{k}\right|}$. O número $R$ é chamado de raio de convergência da série.

A unicidade da transformada é garantida pelo teorema abaixo.

\section{Teorema 5.1:}

a) Seja $f(z)$ uma função analítica para $z \in \mathbb{C}$ tal que $|z|>R$, com $R \geq 0$. Entào existe uma única seqüência $\left\{f_{k}\right\} \in \sigma$ tal que $f(z)=\sum_{k=0}^{\infty} f_{k} z^{-k}$, onde

$$
f_{k}=\frac{1}{2 \pi \mathrm{i}} \oint_{\gamma} f(z) z^{k-1} d z, \quad k=0,1, \ldots,
$$

$\operatorname{com} \gamma=\gamma(\theta)=\rho \mathrm{e}^{\mathrm{i} \theta}, \rho>R$ e $\theta \in[0,2 \pi]$.

b) Se $\sum_{k=0}^{\infty} f_{k} z^{-k}$ converge, para $z \in \mathbb{C}$ tal que $|z|>R$, para algum $R>0$, então existe uma única funçâo analítica $f(z)$ tal que $f(z)=\sum_{k=0}^{\infty} f_{k} z^{-k}$, para $|z|>R$.

Demonstraçào :

a) Se $f(z)$ é uma função analítica para $z \in \mathbb{C}$ tal que $|z|>R$, então a função $F(w)=f\left(\frac{1}{w}\right)$ é analítica para todo $w \in \mathbb{C}$ tal que $|w|<\frac{1}{R}$, onde $z=\frac{1}{w}$. Dessa. forma, $F(w)$ admite um único desenvolvimento de Taylor em torno de $w=0$ dado por

$$
F(w)=\sum_{k=0}^{\infty} a_{k} w w^{k},
$$

onde

$$
a_{k}=\frac{1}{2 \pi \mathrm{i}} \oint_{\gamma_{1}} \frac{F(\zeta)}{\zeta^{k+1}} d \zeta, \text { com } \gamma_{1}=\gamma_{1}(\theta)=\rho \mathrm{e}^{-\mathrm{i} \theta}, \theta \in[0,2 \pi] \text { e } 0<\rho<\frac{1}{R} .
$$

Logo, $f(z)=F\left(\frac{1}{z}\right)=\sum_{k=0}^{\infty} a_{k} z^{-k}$, com

$$
a_{k}=\frac{1}{2 \pi \mathrm{i}} \oint_{\gamma_{1}} \frac{F(\zeta)}{\zeta^{k+1}} d \zeta=\frac{1}{2 \pi \mathrm{i}} \oint_{\gamma} f(z) z^{k-1} d z
$$


onde $\gamma^{\prime}=\gamma(\theta)=\rho \mathrm{e}^{\mathrm{i} \theta}, \theta \in[0,2 \pi]$ e $\rho>\frac{1}{R}$.

b) Se $\sum_{k=0}^{\infty} f_{k} z^{-k}$ é convergente em $\Omega=\{z \in \mathbb{C} /|z|>R\}$, a série $\sum_{k=0}^{\infty} f_{k} w^{k}$ é convergente em $\Omega_{1}=\left\{w \in \mathbb{C} /|w|<\frac{1}{R}\right\}$. Seja $F(w)=\sum_{k=0}^{\infty} f_{k} w^{k}$, para $w \in \Omega_{1} \mathrm{e}$ seja $\gamma$ uma curva fechada contida em $\Omega_{1}$. Como

$$
\oint_{\gamma^{\prime}} F(w) d w=\sum_{k=0}^{\infty} f_{k} \oint_{\gamma} w^{k} d w=0
$$

e $F(w)$ é uma funçào contínua em $\Omega_{1}$, segue, pelo Teorema de Morera, que $F(w)$ é uma função analítica em $\Omega_{1}$. A unicidade de $F(w)$ segue da unicidade das séries de potências na regiào de convergência. Portanto, existe uma única função analítica $f(z)=F\left(\frac{1}{z}\right)=\sum_{k=0}^{\infty} f_{k} z^{-k}$.

O próximo passo é determinar uma condição sobre $\left\{f_{k}\right\} \in \sigma$ tal que $l\left[\left\{f_{k}\right\}\right]$ exista.

Definição 5.3 : A seqüência $\left\{f_{k}\right\} \in \sigma$ é do tipo exponencial se existem $M>0$, $z_{0} \in \mathbb{C}, z_{0} \neq 0$ e $k_{0} \geq 0$ tais que

$$
\left|f_{k}\right|<M\left|z_{0}\right|^{k}, \forall k \geq k_{0} .
$$

Teorema 5.2: Seja $\left\{f_{k}\right\} \in \sigma$. Então, $\left\{f_{k}\right\}$ é de tipo exponencial se, e somente se, $\left\{f_{k}\right\}$ é laurent-transformável.

Demonstração :

$(\Rightarrow)$ Se $\left\{f_{k}\right\}$ é do tipo exponencial, existem $M>0, k_{0} \geq 0$ e $z_{o} \in \mathbb{C}, z_{0} \neq 0$ tais que

$$
\left|f_{k}\right|<M\left|z_{0}\right|^{k} \quad \text {, para } k \geq k_{0}
$$

Então,

$$
l\left[\left\{f_{k}\right\}\right]=\sum_{k=0}^{\infty} f_{k} z^{-k}=\sum_{k=0}^{k_{0}-1} f_{k} z^{-k}+\sum_{k=k_{0}}^{\infty} f_{k} z^{-k}
$$


Para $k \geq k_{0}$, temos

$$
\left|f_{k} z^{-k}\right|=\left|f_{k} z_{0}^{-k}\right|\left|\frac{z}{z_{0}}\right|^{-k}<M\left|\frac{z_{0}}{z}\right|^{k} .
$$

Se $|z|>\left|z_{0}\right|$, a série geométrica $\sum_{k=0}^{\infty} M\left|\frac{z_{0}}{z} .\right|^{k}$ é convergente, logo a série $\sum_{k=k_{0}}^{\infty} f_{k} z^{-k}$ é (absolutamente) convergente. Como $\sum_{k=0}^{k_{0}-1} f_{k} z^{-k}$ tem um número finito de termos, segue que $\sum_{k=0}^{\infty} f_{k} z^{-k}$ é (absolutamente) convergente para todo $z \in \mathbb{C}$ tal que $|z|>\left|z_{0}\right|$. Portanto, $\left\{f_{k}\right\}$ é laurent-transformável.

$(\Leftarrow)$ Suponhamos que $\sum_{k=0}^{\infty} f_{k} z^{-k}$ converge para algum $z_{0} \in \mathbb{C}, z_{0} \neq 0$. Nesse caso, $\left|z_{0}\right|>R$. onde $R$ é o raio de convergência dessa série. Seja $f(z)$ a funçâo analítica. dada por

$$
f(z)=\sum_{k=0}^{\infty} f_{k} z^{-k} \text {, onde }|z|>\left|z_{0}\right| .
$$

Pelo Teorema 5.1, temos

$$
f_{k}=\frac{1}{2 \pi \mathrm{i}} \oint_{\gamma} f(z) z^{k-1} d z
$$

onde $\gamma=\gamma(\theta)=\rho \mathrm{e}^{i \theta}, \theta \in[0,2 \pi]$ e $\rho \geq\left|z_{0}\right|$. Logo,

$$
\begin{aligned}
\left|f_{k}\right| & \leq \frac{1}{2 \pi} \oint_{\gamma}\left|f(z) z^{k-1}\right| d z \\
& \leq \max _{z \in \gamma}|f(z)| \rho^{k} \\
& =M \rho^{k}, \quad k=0,1, \ldots .
\end{aligned}
$$

Vejamos agora algumas propriedades:

Proposição 5.1 (linearidade) Sejam a um escalar em $\mathbb{C},\left\{f_{k}\right\},\left\{g_{k}\right\} \in \sigma, \epsilon$ $f(z)=l\left[\left\{f_{k}\right\}\right]$ para $|z|>r_{1}$ e $g(z)=l\left[\left\{g_{k}\right\}\right]$ para $|z|>r_{2}$ suas respectivas transformadas de Laurent. Então

$$
l\left[\left\{a f_{k}+g_{k}\right\}\right]=a f(z)+g(z), \text { para }|z|>\max \left\{r_{1}, r_{2}\right\} .
$$


Demonstração : Segue da convergência absoluta das séries.

Proposição $\mathbf{5 . 2}$ (translação da seqüência) Sejam $j \in \mathbb{Z}_{+}$fixado, $\left\{f_{k}\right\} \in \sigma e$ $f(z)=l\left[\left\{f_{k}\right\}\right]$. Então

a) $l\left[\left\{f_{k+j}\right\}\right]=z^{j}\left[f(z)-\sum_{i=0}^{j-1} f_{i} z^{-i}\right], k=0,1, \ldots$,
b) $l\left[\left\{f_{k-j}\right\}\right]=z^{-j} f(z), k=0,1, \ldots$

Demonstração :

a)

$$
\begin{aligned}
l\left[\left\{f_{k+j}\right)\right] & =\sum_{k=0}^{\infty} f_{k+j} z^{-k} \\
& =f_{j}+f_{j+1} z^{-1}+\cdots \\
& =\left[\sum_{k=0}^{\infty} f_{k} z^{-k}-f_{0}-f_{1} z^{-1}-\cdots-f_{j-1} z^{-j+1}\right] z^{j} .
\end{aligned}
$$

b) Lembrando que, por convenção, $f_{k}=0$ se $k<0$, temos

$$
l\left[\left\{f_{k-j}\right)\right]=\sum_{k=0}^{\infty} f_{k-j} z^{-k}=f_{0} z^{-j}+f_{1} z^{-j-1}+\cdots=f(z) z^{-j} .
$$

Conhecendo-se a transformada de $\left\{f_{k}\right\}$, podemos analisar o comportamento dessa seqüência pelo teorema abaixo :

Teorema 5.3 (Valor inicial e final) Seja $\left\{f_{k}\right\}$ uma seqüência de $\sigma$.

a) Se $f(z)=l\left[\left\{f_{k}\right\}\right]$ para $|z|>r$, então $f_{0}=\lim _{z \rightarrow \infty} f(z)$.

b) Se $f(z)=l\left[\left\{f_{k}\right\}\right]$ para $|z|>1 e(z-1) f(z)$ é uma funçâo analítica para $z=1$, entâo

$$
\lim _{k \rightarrow \infty} f_{k}=\lim _{z \rightarrow 1}(z-1) f(z)
$$

Demonstração :

a) Segue da definiçào de transformada de Laurent.

b) Das propriedades linearidade e translação, segue que

$$
\begin{aligned}
l\left[\left\{f_{k+1}-f_{k}\right\}\right] & =l\left[\left\{f_{k+1}\right\}\right]-l\left[\left\{f_{k}\right\}\right]=z\left[f(z)-f_{0}\right]-f(z) \\
& =(z-1) f(z)-f_{0} .
\end{aligned}
$$


Por outro lado,

$$
l\left[\left\{f_{k+1}-f_{k}\right\}\right]=\sum_{k=0}^{\infty}\left(f_{k+1}-f_{k}\right) z^{-k}=\lim _{n \rightarrow \infty} \sum_{k=0}^{n}\left(f_{k+1}-f_{k}\right) z^{-k} .
$$

Considerando o limite das expressões acima, $\operatorname{com} z$ tendendo a 1 , temos

$$
\begin{aligned}
& \lim _{z \rightarrow 1} l\left[\left\{f_{k+1}-f_{k}\right\}\right]=\lim _{n \rightarrow \infty} \sum_{k=0}^{n}\left(f_{k+1}-f_{k}\right)=\lim _{n \rightarrow \infty}\left(f_{n+1}-f_{0}\right) \\
& \lim _{z \rightarrow 1} l\left[\left\{f_{k+1}-f_{k}\right\}\right]=\lim _{z \rightarrow 1}(z-1)\left[f(z)-f_{0}\right] .
\end{aligned}
$$

Logo,

$$
\lim _{z \rightarrow 1}(z-1) f(z)=\lim _{n \rightarrow \infty} f_{n+1}=\lim _{n \rightarrow \infty} f_{n}
$$

Vamos definir convolução de duas seqüências e verificar que a transformada da convoluçâo de duas seqüências corresponde ao produto de suas respectivas transformadas.

Definição 5.4 : Sejam $\left\{f_{k}\right\},\left\{g_{k}\right\} \in \sigma$. A convolução das seqüências $\left\{f_{k}\right\}$ e $\left\{g_{k}\right\}$ é a seqüência dada por :

$$
\left\{f_{k}\right\} *\left\{g_{k}\right\}=\left\{\sum_{j=0}^{k} f_{j} g_{k-j}\right\}_{k=0}^{\infty} .
$$

Por simplicidade, denotaremos por $f_{k} * g_{k}=\sum_{j=0}^{k} f_{j} g_{k-j}$.

A convolução é uma operaçào que satisfaz as seguintes propriedades :

a) comutativa : $f_{k} * g_{k}=g_{k} * f_{k}$,

b) associativa : $f_{k} *\left(g_{k} * h_{k}\right)=\left(f_{k} * g_{k}\right) * h_{k}$,

c) distributiva em relaçào à adiçào : $f_{k} *\left(g_{k}+h_{k}\right)=f_{k} * g_{k}+f_{k} * h_{k}$. 
Proposição 5.3 : Sejam $\left\{f_{k}\right\},\left\{g_{k}\right\} \in \sigma, f(z)=l\left[\left\{f_{k}\right\}\right]$, para $|z|>r_{1}$ e $g(z)=$ $l\left[\left\{g_{k}\right\}\right]$, para $|z|>r_{2}$. Então $l\left[f_{k} * g_{k}\right]=f(z) g(z)$, para $|z|>\max \left\{r_{1}, r_{2}\right\}$.

Demonstração: Para $|z|>\max \left\{r_{1}, r_{2}\right\}$, temos

$$
f(z) g(z)=\sum_{k=0}^{\infty} f_{k} z^{-k} \sum_{k=0}^{\infty} g_{k} z^{-k}=\sum_{k=0}^{\infty} \sum_{j=0}^{k} f_{k} g_{k-j} z^{-k}=l\left[f_{k} * g_{k}\right] .
$$

Em particular, se $f(z)=g(z)$, temos $f^{2}(z)=l\left[f_{k} * f_{k}\right]$.

Dada $f(z)$ uma função analítica, o processo de obter uma seqüência $\left\{f_{k}\right\} \in \sigma$ tal que $f(z)=\sum_{k=0}^{\infty} f_{k} z^{-k}$ é chamado de transformada inversa de Laurent. Simbolicamente, queremos obter $\left\{f_{k}\right\}=l^{-1}[f(z)]$. Apresentaremos, a seguir, alguns métodos para obtenção da inversa da transformada de Laurent, sendo que a unicidade decorre do Teorema 5.1.

Uma vez que a transformada de Laurent matricial da solução de uma equação de diferença matricial linear é uma matriz cujos elementos são funções racionais, como veremos na Seção 5.3, vamos nos restringir ao estudo da transformada inversa de funções racionais.

O método mais simples consiste em usar uma tabela de seqüências com as respectivas transformadas. O uso da tabela deve ser criteriosa, já que em geral a região de convergência nào é especificada. Uma lista de transformadas de Laurent pode ser encontrada em [9].

Método numérico da transformada inversa : Se $f(z)$ é uma função racional, podemos determinar os coeficientes $f_{k}$ através da divisão de polinômios, como se segue. Se $f(z)$ é da forma :

$$
\frac{p(z)}{q(z)}=f(z)=\frac{a_{0} z^{n}+a_{1} z^{n-1}+\cdots+a_{n}}{z^{n}+b_{1} z^{n-1}+\cdots+b_{n}},
$$


ao dividirmos $p(z)$ por $q(z)$, obtemos uma série com expoentes negativos em $z$, ou seja :

$$
\frac{p(z)}{q(z)}=a_{0}+\left(a_{1}-a_{0} b_{1}\right) z^{-1}+\cdots .
$$

Se $f(z)=\sum_{k=0}^{\infty} f_{k} z^{-k}$, temos

$$
\left\{\begin{array}{l}
f_{0}=a_{0} \\
f_{1}=a_{1}-b_{1} a_{0}=a_{1}-b_{1} f_{0} \\
f_{2}=a_{2}-b_{1}\left(a_{1}-b_{1} a_{0}\right)-b_{2} a_{0}=a_{2}-b_{1} f_{1}-b_{2} f_{0} \\
\quad \vdots \\
f_{k}=a_{k}-\sum_{i+j=k} b_{i} f_{j},
\end{array}\right.
$$

sendo $a_{k}=0$ para $k>n$.

Método dos Resíduos : Consiste na aplicação do teorema integral de Cauchy. Lembramos que o resíduo de uma funçâo $f(z)$ em um pólo $z_{0}$ de ordem $\nu$ é o termo $b_{1}$ do desenvolvimento de Laurent de $f(z)$ em torno desse ponto $z_{0}$ :

$$
f(z)=\sum_{k=0}^{\infty} a_{k}\left(z-z_{0}\right)^{k}+\sum_{k=1}^{\nu} b_{k}\left(z-z_{0}\right)^{-k},
$$

onde a parte principal dessa série tem um número finito de termos, conhecidos como fraçòes parciais.

Teorema 5.4: Se $f(z)$ é uma função racional, entâo existe uma única seqüência $\left\{f_{k}\right\} \in \sigma$ tal que $f(z)=\sum_{k=1}^{\infty} f_{k} z^{-k}$, onde

$$
f_{k}=\sum_{t=1}^{q} \operatorname{res}\left(f(z) z^{k-1}, z_{t}\right),
$$

onde q é o número de pólos distintos de $f(z) z^{k-1}$ e res $\left(f(z) z^{k-1}, z_{t}\right)$ denota o residuo de $f(z) z^{k-1}$ no pólo $z_{t}$. 
Demonstração : Segue do Teorema 5.1 e do Teorema dos Resíduos que

$$
f_{k}=\frac{1}{2 \pi i} \oint_{\gamma} f(z) z^{k-1} d z=\sum_{t=1}^{q} \operatorname{res}\left(f(z) z^{k-1}, z_{t}\right),
$$

onde $\gamma=\gamma(\theta)=\rho \mathrm{e}^{\mathrm{i} \theta}, \theta \in[0,2 \pi]$ e $\rho>R=\max _{1 \leq t \leq d}\left\{\left|z_{t}\right|\right\}$

Vejamos como determinar o resíduo de uma funçào em um pólo.

Teorema 5.5: Seja h.(z) uma função analítica em uma vizinhança de $z_{0} \in \mathbb{C}$, exceto em $z=z_{0}$. Se $z_{0}$ é um pólo de ordem $\nu \geq 1$ de $h(z)$, então

$$
\operatorname{res}\left(h(z), z_{0}\right)=\frac{1}{(\nu-1) !} \lim _{z \rightarrow z_{0}} \frac{d^{\nu-1}}{d z^{\nu-1}}\left[\left(z-z_{0}\right)^{\nu} h(z)\right] \text {. }
$$

Em particular, se $\nu=1$, temos

$$
\operatorname{res}\left(h(z), z_{0}\right)=\lim _{z \rightarrow z_{0}}\left[\left(z-z_{0}\right) h(z)\right] .
$$

Demonstração : Seja $g(z)$ uma função analítica em $z=z_{0}$, com $g\left(z_{0}\right) \neq 0$ tal que $g(z)=h(z)\left(z-z_{0}\right)^{\nu}$. Pela equaçào (5.1), temos que

$$
g(z)=h(z)\left(z-z_{0}\right)^{\nu}=\sum_{k=0}^{\infty} a_{k}\left(z-z_{0}\right)^{k+\nu}+b_{1}\left(z-z_{0}\right)^{\nu-1}+\cdots+b_{\nu}
$$

Para determinar $b_{1}$, derivamos a expressão acima $\nu-1$ vezes :

$$
\frac{d^{\nu-1}}{d z^{\nu-1}}\left[\left(z-z_{0}\right)^{\nu} h(z)\right]=(\nu-1) ! b_{1}+\sum_{k=0}^{\infty} a_{k}(k+\nu-1) !\left(z-z_{0}\right)^{k},
$$

e fazemos o limite para $z$ tendendo a $z_{0}$ :

$$
b_{1}=\operatorname{res}\left(h(\tilde{z}), z_{0}\right)=\frac{1}{(\nu-1) !} \lim _{z \rightarrow z_{0}} \frac{d^{\nu-1}}{d z^{\nu-1}}\left[\left(z-z_{0}\right)^{\nu} h(z)\right] .
$$


Observação 5.1 : Seja $h(z)$ uma função racional com pólos de ordem $\nu_{1}, \ldots, \nu_{q}$ em $z_{1}, \ldots, z_{q}$, respectivamente. Podemos relacionar o resíduo de $h(z)$ com as fraçôes parciais da seguinte forma : se a parte principal do desenvolvimento em Laurent de $h(z)$ em torno de $z_{t}$ é dada por

$$
P\left(z, z_{t}\right)=\sum_{u=1}^{\nu_{t}} \frac{b_{u, t}}{\left(z-z_{t}\right)^{u}}
$$

com $b_{u, t} \in \mathbb{C}$. temos que

$$
h(z)=\sum_{t=1}^{q} P\left(z, z_{t}\right)+g(z)
$$

onde $g(\tilde{z})$ é uma função analítica em $\mathbb{C}$. Pela definição de resíduo, segue que

$$
\sum_{t=1}^{q} \operatorname{res}\left(h(z), z_{t}\right)=\sum_{t=1}^{q} b_{1, t}
$$

com $b_{1, t}$ como em (5.2).

Consideramos agora $h(z)=f(z) z^{k-1}$, para $k \geq 0$ fixado, onde $f(z)$ é uma função racional. Pelo Teorema 5.5, segue que

$$
\operatorname{res}\left(f(z) z^{k-1}, z_{t}\right)=\frac{1}{\left(\nu_{t}-1\right) !} \lim _{z \rightarrow z_{t}} \frac{d^{\nu_{t}-1}}{d z^{\nu_{t}-1}}\left[\left(z-z_{t}\right)^{\nu_{t}} f(z) z^{k-1}\right] \text {. }
$$

Se usamos a regra de Leibnitz para derivação, podemos escrever a fórmula acima como :

$$
\operatorname{res}\left(f(z) z^{k-1}, z_{t}\right)=\sum_{u=1}^{\nu_{t}} \frac{1}{\left(\nu_{t}-u\right) !}\left(\begin{array}{l}
k-1 \\
u-1
\end{array}\right) z_{t}^{k-u} \frac{d^{\nu_{t}-u}}{d z^{\nu_{t}-u}}\left[\left(z-z_{t}\right)^{\nu_{t}} f(z)\right]_{z=z_{t}} .
$$

Em particular, se $z_{0}$ é um pólo simples de $f(z)$ uma função racional da forma $\frac{p(z)}{q(z)}$, onde $p(z)$ e $q(z)$ sâo polinômios tais que o grau de $p(z)$ é menor que o grau de $q(z)$, temos

$$
\operatorname{res}\left(f(z) z^{k-1}, z_{0}\right)=\lim _{z \rightarrow z_{0}}\left[\left(z-z_{0}\right) f(z) z^{k-1}\right]=\frac{p\left(z_{0}\right)}{q^{\prime}\left(z_{0}\right)} z_{0}^{k-1}
$$


Observamos que um cuidado especial deve ser tomado em relação à existência de pólos de $f(z) z^{k-1}$ em $z=0$; por exemplo : se $z=0$ é um pólo de $f(z)$ de multiplicidade $\nu$, então (5.3) permite obter os termos $f_{k}$ onde $k \geq \nu+1$, pois nesse caso, $z=0$ é um zero de $f(z) z^{k-1}$ de multiplicidade $(k-\nu-1)$, para $0 \leq k \leq \nu$. Os $\nu$ primeiros termos de $\left\{f_{k}\right\}$ podem ser obtidos através do método numérico da. transformada inversa citado acima ou calculando o resíduo de $f(z) z^{k-1}$ em $z=0$ separadamente, como na Proposição abaixo.

Proposição 5.4: Seja $f(z)$ uma função racional que admite pólos $z_{1}, \ldots, z_{q}$ de ordens $\nu_{1}, \ldots, \nu_{q}$, respectivamente, onde $\nu_{i} \geq 1$. Se $z_{1}=0$, entâo

$$
f_{k}=\left\{\begin{array}{l}
\sum_{t=2}^{q} \operatorname{res}\left(f(z) z^{k-1}, z_{t}\right)+\operatorname{res}\left(f(z) z^{k-1}, 0\right), \quad \text { para } 0 \leq k \leq \nu_{1} \\
\sum_{t=2}^{q} \operatorname{res}\left(f(z) z^{k-1}, z_{t}\right), \quad \text { para } k \geq \nu_{1}+1
\end{array}\right.
$$

Demonstração: Basta observar que $z=0$ é um pólo de ordem $\left(\nu_{1}+1-k\right)$ de $f(z) z^{k-1}$, para $0 \leq k \leq \nu_{1}$.

Por outro lado, se $z=0$ não é nem pólo nem zero de $f(z)$, os termos $f_{k}, k \geq 1$ podem ser obtidos pelo Teorema 5.5 e $f_{0}$ pode obtido do Teorema 5.3 .

O teorema seguinte permite determinar $\left\{f_{k}\right\}$ sem utilizar os pólos de $f(z)$.

Teorema 5.6: Seja $f(z)$ uma funçâo analítica para $z \in \mathbb{C}$ tal que $|z|>R$. Então existe uma única seqüência $\left\{f_{k}\right\} \in \sigma$ tal que $\sum_{k=0}^{\infty} f_{k} z^{-k}=f(z)$, onde

$$
f_{k}=\frac{1}{k !}\left[\frac{d^{k}}{d w^{k}} f\left(\frac{1}{w}\right)\right]_{w=0}, \quad k=0,1, \ldots .
$$

Demonstração: A funçào $f\left(\frac{1}{w}\right)$ é analítica em $w=0$ e sua expansão em série de Taylor, em torno de $w=0$, tem os coeficientes como indicado acima. 


\subsection{Transformada de Laurent - Caso matricial}

Nesta seçâo, apresentamos uma extensão da transformada de Laurent às seqüências de matrizes.

Definição 5.5 : Seja $\left\{Y_{k}\right\} \in \mathcal{M}$. Chamamos a soma $\sum_{k=0}^{\infty} Y_{k}$ de série de matrizes, onde $Y_{k}$ é o termo geral. Seja $S_{n}=\sum_{k=0}^{n-1} Y_{k}$ a seqüência das somas parciais. Dizemos que a série de matrizes $\sum_{k=0}^{\infty} Y_{k}$ converge se a seqüência das somas parciais $S_{n}$ converge, ou seja, se existir $\lim _{n \rightarrow \infty} S_{n}$ e, nesse caso, indicamos

$$
\sum_{k=0}^{\infty} Y_{k}=\lim _{n \rightarrow \infty} S_{n}
$$

como a soma da série.

Para $k$ fixado, denotaremos o (ij)-ésimo elemento de $Y_{k}$ por $y_{i j}^{(k)}$. A série de matrizes $\sum_{k=0}^{\infty} Y_{k}$ representa $m^{2}$ séries infinitas da forma $\sum_{k=0}^{\infty} y_{i j}^{(k)}$ onde $1 \leq i, j \leq m$.

A transformada de Laurent matricial é uma aplicação que associa cada seqüência $\left\{Y_{k}\right\}$ de $\mathcal{M}$ a uma matriz $F(z), \operatorname{com} z \in \mathbb{C}$, e é denotada por :

$$
\mathcal{L}\left[\left\{Y_{k}\right\}\right]=F(z) \text { ou simplesmente } \mathcal{L}\left[Y_{k}\right]=F(z)
$$

Definição 5.6 : Seja $\left\{Y_{k}\right\} \in \mathcal{M}$. A transformada de Laurent matricial de $\left\{Y_{k}\right\}$ é a matriz $F(z)$ definida por $F(z)=\sum_{k=0}^{\infty} Y_{k} z^{-k}$, onde $z \in \mathbb{C}$ são os valores para os quais a série converge.

Seja $\rho_{i j}$ o raio de convergência da série $\sum_{k=0}^{\infty} y_{i j}^{(k)} z^{-k}$, para $1 \leq i, j \leq m$. Chamamos $R=\max _{1 \leq i, j \leq m}\left\{\rho_{i j}\right\}$ de raio de convergência da série de matrizes $\sum_{k=0}^{\infty} Y_{k} z^{-k}$. 
Estamos supondo que as seqüências $\left\{y_{i j}^{(k)}\right\}$ de $\left\{Y_{k}\right\}$ são do tipo exponencial, pois como já vimos na seção 1 deste capítulo, essa condição garante a convergência. absoluta da série dada pela transformada de Laurent.

Podemos enunciar um teorema análogo ao Teorema 5.1 para a transformada de Laurent matricial.

Teorema 5.7: Sejam $\left\{Y_{k}\right\} \in \mathcal{M}$ e $F(z) \in \mathbb{C}^{m \times m}$.

a) Se $\sum_{k=0}^{\infty} Y_{k} z^{-k}$ converge para $|z|>R$, então existe uma única função $F(z)$ tal que $F(z)=\sum_{k=0}^{\infty} Y_{k} z^{-k}$

b) Se os elementos de $F(z)$ são funçôes racionais definidas em $|z|>R$, entâo existe uma única seqüência $\left\{Y_{k}\right\}$ tal que $F(z)=\sum_{k=0}^{\infty} Y_{k} z^{-k}$.

Demonstração : Basta aplicar o Teorema (5.1) a cada elemento das matrizes.

Definição 5.7 : Dizemos que $\left\{Y_{k}\right\} \in \mathcal{M}$ é laurent-transformável se cada elemento $\left\{y_{i j}^{(k)}\right\}$ é laurent-transformável.

Vamos apresentar a seguir algumas propriedades desta transformada :

Proposição 5.5 : Seja A uma matriz constante em $\mathbb{C}^{m \times m}$, $\epsilon$ sejam as matrizes $F(z)=\mathcal{L}\left[\left\{Y_{k}\right\}\right], \operatorname{para}|z|>R_{1}$ e $G(z)=\mathcal{L}\left[\left\{W_{k}\right\}\right]$ para $|z|>R_{2}$. Entâo :

$$
\mathcal{L}\left[\left\{A Y_{k}+W_{k}\right\}\right]=A F(z)+G(z), \quad \text { para }|z|>\max \left\{R_{1}, R_{2}\right\} .
$$

Demonstraçâo :

$$
\mathcal{L}\left[\left\{Y_{k}+W_{k}\right\}\right]=\sum_{k=0}^{\infty}\left(A Y_{k}+W_{k}\right) z^{-k}=A \sum_{k=0}^{\infty} Y_{k} z^{-k}+\sum_{k=0}^{\infty} W_{k} z^{-k},
$$

para $|z|>\max \left\{R_{1}, R_{2}\right\}$. 
Proposição 5.6 (translação da seqüência) Sejam $j \in \mathbb{Z}_{+}$fixado, $\left\{Y_{k}\right\} \in \mathcal{M} e$ $F(z)=\sum_{k=0}^{\infty} Y_{k} z^{-k}$ a transformada de Laurent matricial de $\left\{Y_{k}\right\}$. Então

a) $\mathcal{L}\left[\left\{Y_{k+j}\right\}\right]=z^{j}\left[F(z)-\sum_{i=0}^{j-1} Y_{i} z^{-i}\right], k=0,1, \ldots$,

b) $\mathcal{L}\left[\left\{Y_{k-j}\right\}\right]=z^{-j} F(z), k=0,1, \ldots$.

Demonstração :

a)

$$
\begin{aligned}
\mathcal{L}\left[\left\{Y_{k+j}\right\}\right] & =\sum_{k=0}^{\infty} Y_{k+j} z^{-k} \\
& =Y_{j}+Y_{j+1} z^{-1}+\cdots \\
& =\left[\sum_{k=0}^{\infty} Y_{k} z^{-k}-Y_{0}-Y_{1} z^{-1}-\cdots-Y_{j-1} z^{-j+1}\right] z^{j}
\end{aligned}
$$

b) Basta lembrar que $Y_{k}=0$ se $k<0$.

Vamos definir a convolução entre seqüências de matrizes e verificar que a transformada de Laurent matricial aplicada em uma convolução de $\left\{Y_{k}\right\}$ e $\left\{W_{k}\right\}$ resulta no produto das transformadas matriciais de $\left\{Y_{k}\right\}$ e $\left\{W_{k}\right\}$.

Definição 5.8: Sejam $\left\{Y_{k}\right\}$ e $\left\{W_{k}\right\} \in \mathcal{M}$. A convolução das seqüências $\left\{Y_{k}\right\}$ e $\left\{W_{k}\right\}$ é a seqüência $\left\{U_{k}\right\}$ dada por :

$$
U_{k}=\sum_{j=0}^{k} Y_{j} W_{k-j},
$$

e é denotada por $\left\{Y_{k}\right\} *\left\{W_{k}\right\}$ ou simplesmente por $Y_{k} * W_{k}$.

Observamos que a convolução de seqüências de matrizes não é comutativa.

Proposição 5.7: Sejam $\left\{Y_{k}\right\},\left\{W_{k}\right\} \in \mathcal{M}, F(z)=\mathcal{L}\left[\left\{Y_{k}\right\}\right]$ para $|z|>R_{1} e$ $G(z)=\mathcal{L}\left[\left\{W_{k}\right\}\right]$ para $|z|>R_{2}$. Entào $\mathcal{L}\left[Y_{k} * W_{k}\right]=F(z) G(z)$ para $|z|>$ $\max \left\{R_{1}, R_{2}\right\}$. 
Demonstração: $\operatorname{Se}|z|>\max \left\{R_{1}, R_{2}\right\}$, temos

$$
F(z) G(z)=\sum_{k=0}^{\infty} Y_{k} z^{-k} \sum_{k=0}^{\infty} W_{k} z^{-k}=\sum_{k=0}^{\infty} U_{k} z^{-k},
$$

com $U_{k}=\sum_{j=0}^{k} Y_{k} W_{k-j}$. Logo, pela unicidade da transformada de Laurent, segue que

$$
\mathcal{L}\left[Y_{k} * W_{k}\right]=F(z) G(z)
$$

Vamos apresentar agora algumas transformadas de Laurent matriciais. Para. tanto, enunciaremos a seguinte :

Definição 5.9 : Sejam $\lambda_{1}, \ldots, \lambda_{m}$ os valores próprios de $A \in \mathbb{C}^{m \times m}$. Chamamos de raio espectral de $A$ ao número $\rho(A)=\max _{1 \leq i \leq m}\left|\lambda_{i}\right|$.

Lema 5.1 ([14]) Seja $A \in \mathbb{C}^{m \times m}$. Entâo $\lim _{k \rightarrow \infty} A^{k}=0 \Leftrightarrow \rho(A)<1$.

Proposição 5.8: Sejam $A, B \in \mathbb{C}^{m \times m}$. Então :

a) $\mathcal{L}[\{A\}]=\frac{z}{z-1} A$, se $|z|>1$;

b) $\mathcal{L}\left[\left\{A^{k} B\right\}\right]=z(z I-A)^{-1} B$, se $\rho\left(A z^{-1}\right)<1$ e z não é um valor próprio de $A$.

\section{Demonstraçâo :}

a) $\mathcal{L}[\{A\}]=\sum_{k=0}^{\infty} A z^{-k}=A \sum_{k=0}^{\infty} z^{-k}=A \frac{z}{z-1}$, se $|z|>1$.

b) Como $\mathcal{L}\left[\left(A^{k} B\right)\right]=\sum_{k=0}^{\infty} A^{k} B z^{-k}=\sum_{k=0}^{\infty} A^{k} z^{-k} B$, a seqüência das somas parciais é dada por

$$
S_{n}=\sum_{k=0}^{n-1} A^{k} z^{-k} B=\left[I+A z^{-1}+\cdots+A^{n-1} z^{-n+1}\right] B .
$$

Então,

$$
S_{n}-A z^{-1} S_{n}=\left[I-A^{n} z^{-n}\right] B .
$$


Se $z$ não é um valor próprio de $A$, então $\left(I-A z^{-1}\right)$ é não-singular e

$$
S_{n}=\left(I-A z^{-1}\right)^{-1}\left(I-A^{n} z^{-n}\right) B .
$$

Logo,

$$
\lim _{n \rightarrow \infty} S_{n}=\lim _{n \rightarrow \infty}\left[\left(I-A z^{-1}\right)^{-1} B-A^{n} z^{-n} B\right] .
$$

Como $\rho\left(A z^{-1}\right)<1$, pelo lema anterior, temos que $\lim _{n \rightarrow \infty}\left(A z^{-1}\right)^{n}=0$. Portanto,

$$
\sum_{k=0}^{\infty} A^{k} B z^{-k}=\lim _{n \rightarrow \infty} S_{n}=\left(I-A z^{-1}\right)^{-1} B=z(z I-A)^{-1} B
$$

De modo análogo, segue que $\mathcal{L}\left[\left\{B A^{k}\right\}\right]=z B(z I-A)^{-1}$, se $\rho\left(A z^{-1}\right)<1$ e $z$ não é um valor próprio de $A$. Observemos que os itens (a) e (b) acima mostram como sào as transformadas de Laurent matricial de uma seqüência constante bem como de uma seqüência de potências.

\subsection{Transformada de Laurent matricial da solução de uma equação de diferença matricial linear}

Nesta seçâo, veremos que a transformada de Laurent matricial da solução de um problema de valores iniciais envolvendo uma equaçào de diferença matricial linear de ordem $n$ é uma função racional.

Consideremos a soluçào $\left\{Y_{k}\right\}$ do seguinte problema :

$$
Y_{k+n}+A_{1} Y_{k+n-1}+\cdots+A_{n} Y_{k}=B_{k}, k=0,1, \ldots,
$$


com as condições iniciais $Y_{0}=D_{0}, \ldots, Y_{n-1}=D_{n-1}$, onde $D_{j} \in \mathbb{C}^{m \times m}, j=0, \ldots, n-1$. Vamos supor que $\left\{Y_{k}\right\}$ é laurent-transformável. Seja $F(z)=\mathcal{L}\left[\left\{Y_{k}\right\}\right]$. Aplicando a. transformada de Laurent na equação acima, temos:

$$
\mathcal{L}\left[\left\{Y_{k+n}+\sum_{j=1}^{n} Y_{k+n-j}\right\}\right]=\mathcal{L}\left[\left\{B_{k}\right\}\right], k=0,1, \ldots
$$

Pelas Proposições 5.5 e 5.6, segue-se que

$$
z^{n}\left[F(z)-\sum_{i=0}^{n-1} Y_{i} z^{-i}\right]+\cdots+A_{n-1} z\left[F(z)-Y_{0}\right]+A_{n} F(z)=H(z),
$$

sendo $H(z)=\mathcal{L}\left[\left\{B_{k}\right\}\right]$. Resolvendo essa equaçào algébrica em $F(z)$ e usando as condiçòes iniciais do problema., temos :

$$
\begin{gathered}
{\left[z^{n} I+A_{1} \tilde{z}^{n-1}+\cdots+A_{n}\right] F(z)=z^{n}\left(\sum_{i=0}^{n-1} D_{i} z^{-i}\right)+A_{1} z^{n-1}\left(\sum_{i=0}^{n-2} D_{i} z^{-i}\right)+\cdots} \\
+A_{n-1} D_{0} z+H(z)
\end{gathered}
$$

Vamos supor que $z \in \mathbb{C}$ nào é uma raiz latente da $\lambda$-matriz $p(\lambda)=\lambda^{n} I+$ $\sum_{i=1}^{n} A_{i} \lambda^{n-i}$. Nesse caso, para $\lambda=z, \operatorname{det} p(z) \neq 0$ e, como foi observado na Seçào 2.1, podemos escrever

$$
p^{-1}(z)=\frac{\operatorname{adj} p(z)}{\operatorname{det} p(\lambda)}=\frac{a(z)}{\operatorname{det} p(z)}
$$

Logo, resolvendo a equaçâo (5.5) em $F(z)$, temos :

$$
F(z)=p^{-1}(z)[s(z)+H(z)]
$$

onde $s(z)=z^{n} D_{0}+\sum_{w=1}^{n-1} E_{w} z^{n-1}$ é uma $\lambda$-matriz de grau $n \operatorname{com} E_{w}=\sum_{i+j=w} A_{i} D_{j} \mathrm{e}$ $A_{0}=I$.

Veremos, a seguir, como definir e usar a transformada inversa de Laurent matricial para determinar a soluçâo $\left\{Y_{k}\right\}$. 


\subsection{Decomposição em Frações Parciais e a Inversa da Transformada de Laurent matricial}

Seja $F(z)$ uma matriz em $\mathbb{C}^{m \times m}$ cujos elementos $F_{i j}(z)$ são funções analíticas para $|z|>R$. Usaremos o símbolo $\mathcal{L}^{-1}[F(z)]$ para denotar uma seqüência. $\left\{Y_{k}\right\} \in \mathcal{M}$ cuja transformada de Laurent matricial é $F(z)$. Logo, se $\mathcal{L}\left[\left\{Y_{k}\right\}\right]=F(z)$, chamamos $\left\{Y_{k}\right\}=\mathcal{L}^{-1}[F(z)]$ de transformada de Laurent inversa de $F(z)$.

Conforme a Seçào 5.3, a transformada de Laurent matricial de uma soluçâo de uma equação de diferença matricial linear é uma matriz cujos elementos são funções racionais. Uma maneira de determinar a inversa desta transformada consiste na decomposiçâo em fraçôes parciais da inversa de uma lambda-matriz. Vários autores apresentaram um estudo detalhado sobre essa decomposição, como Frazer [3] e Lancaster [12].

Seja $g(\lambda) \in \mathbb{C}^{m \times m}$ uma $\lambda$-matriz arbitrária, $p(\lambda)=\lambda^{n} I+\sum_{j=1}^{n} A_{j} z^{n-j}$ e $a(\lambda)$ a. matriz adjunta de $p(\lambda)$. Nesta seção, consideramos a matriz

$$
U(\lambda)=\frac{a(\lambda) g(\lambda)}{\operatorname{det} p(\lambda)} .
$$

Cada elemento de $U^{\prime}(\lambda)$ é uma função racional cujo denominador sempre é $\operatorname{det} p(\lambda)$. Se grau $a(\lambda) g(\lambda) \geq$ grau $(\operatorname{det} p(\lambda))=m n$, entào para $1 \leq i, j \leq m$,

$$
U_{i j}(\lambda)=q_{i, j}(\lambda)+\frac{r_{i j}(\lambda)}{\operatorname{det} p(\lambda)},
$$

onde $q_{i j}(\lambda)$ e $r_{i j}(\lambda)$ são polinômios escalares e grau $r_{i j}<m n$. Se grau $a(\lambda) g(\lambda)<$ $m n$, então $q_{i j}(\lambda)=0$.

Em termos de matrizes, escrevemos que

$$
U(\lambda)=q(\lambda)+\frac{r(\lambda)}{\operatorname{det} p(\lambda)}
$$


onde $q(\lambda)$ e $r(\lambda)$ são $\lambda$-matrizes cujos elementos sâo $q_{i j}(\lambda)$ e $r_{i j}(\lambda)$, respectivamente. Teorema 5.8 : Sejam $p(\lambda), a(\lambda)$ e $g(\lambda) \lambda$-matrizes como descritas acima. Então

$$
U(\lambda)=\frac{a(\lambda) g(\lambda)}{\operatorname{det} p(\lambda)}
$$

admite uma decomposição em fraçôes parciais.

Demonstração: Já vimos que

$$
U(\lambda)=q(\lambda)+\frac{r(\lambda)}{\operatorname{det} p(\lambda)} .
$$

Sejam $\lambda_{1}, \ldots, \lambda_{q}$ raizes latentes de $p(\lambda)$ de multiplicidades $\nu_{1}, \ldots, \nu_{q}$, respectivamente, para $1 \leq \nu_{t} \leq m n$ e $\sum_{t=1}^{q} \nu_{t}=m n$.

Para cada raiz latente $\lambda_{s}$ de multiplicidade $\nu_{s}$ associamos uma soma de $\nu_{s}$ frações parciais da forma.

$$
\sum_{u=1}^{\nu_{s}} \frac{W_{u, s}}{\left(\lambda-\lambda_{s}\right)^{u}}
$$

onde $B_{u, s} \in \mathbb{C}^{m \times m}, u=1, \ldots, \nu_{s}$, são os coeficientes a determinar.

Logo, podemos escrever

$$
\frac{a(\lambda) g(\lambda)}{\operatorname{det} p(\lambda)}=q(\lambda)+\sum_{u=1}^{\nu_{1}} \frac{W_{u, 1}}{\left(\lambda-\lambda_{1}\right)^{u}}+\cdots+\sum_{u=1}^{\nu_{q}} \frac{W_{u, q}}{\left(\lambda-\lambda_{q}\right)^{u}} .
$$

Para determinar os coeficientes relativos à raiz $\lambda_{s}, 1 \leq s \leq q$, multiplicamos a equação acima por $\left(\lambda-\lambda_{s}\right)^{\nu_{s}}$, obtendo :

$$
\begin{aligned}
\frac{a(\lambda) g(\lambda)}{\prod_{w=1, u \neq s}^{q}\left(\lambda-\lambda_{w}\right)^{\nu_{w}}}=(\lambda & \left.-\lambda_{s}\right)^{\nu_{s}}\left[a(\lambda)+\sum_{\substack{t=1 \\
t \neq s}}^{q} \sum_{u=1}^{\nu_{t}} \frac{W_{u, t}}{\left(\lambda-\lambda_{t}\right)^{u}}\right] \\
& +W_{1, s}\left(\lambda-\lambda_{s}\right)^{\nu_{s}-1}+\cdots+W_{\nu_{s}-1, s}\left(\lambda-\lambda_{s}\right)+W_{\nu_{s}, s} .
\end{aligned}
$$


Derivando sucessivamente a expressâo acima em relação a $\lambda$ e fazendo $\lambda=\lambda_{s}$, obtemos :

$$
W_{u, s}=\frac{1}{\left(\nu_{s}-u\right) !} \frac{d^{\nu_{s}-u}}{d \lambda^{\nu_{s}-u}}\left[\frac{a(\lambda) g(\lambda)}{\prod_{\substack{w=1 \\ w \neq s}}^{q}\left(\lambda-\lambda_{w}\right)^{\nu_{w}}}\right]_{\lambda=\lambda_{s}},
$$

onde $u=1, \ldots, \nu_{s}$.

Portanto,

$$
U(\lambda)=q(\lambda)+\sum_{t=1}^{q} \sum_{u=1}^{\nu_{t}} \frac{W_{u, t}}{\left(\lambda-\lambda_{t}\right)^{u}},
$$

onde $W_{u, t}$ é da.do por (5.7).

Observação 5.2 : Se $\nu_{s}=1$, entâo para a raiz latente $\lambda_{s}$ associamos uma única fraçâo parcial $B_{1, s}\left(\lambda-\lambda_{s}\right)^{-1}$, onde

$$
W_{s}=W_{1, s}=\frac{1}{\frac{d}{d \lambda}[\operatorname{det} p(\lambda)]_{\lambda=\lambda_{s}}} a\left(\lambda_{s}\right) g\left(\lambda_{s}\right) .
$$

Observação 5.3: Se $g(\lambda)=I$ e $a(\lambda)=\operatorname{adj} p(\lambda)$ então

$$
U(\lambda)=\frac{a(\lambda)}{\operatorname{det} p(\lambda)}=p^{-1}(\lambda) .
$$

Nesse caso, $p^{-1}(\lambda)$ admite uma decomposição em fraçôes parciais como descrita no teorema acima, onde $Q(\lambda)=0$ para todo $\lambda$, pois o grau de adj $p(\lambda)$ é igual a $n(m-1)<m . n$.

\subsection{Aplicação}

Voltando ao exemplo da. Seçào 5.3, seja $\left\{Y_{k}\right\} \in \mathcal{M}$ a solução do problema :

$$
Y_{k+n}+A_{1} Y_{k+n-1}+\cdots+A_{n} Y_{k}=B_{k}, \quad k=0,1, \ldots,
$$


com as condiçôes iniciais $Y_{0}=D_{0}, \ldots, Y_{n-1}=D_{n-1}$, onde $D_{j} \in \mathbb{C}^{m \times m}, j=0, \ldots, n-1 \mathrm{e}$ seja $F(z)$ a transformada de Laurent matricial de $\left\{Y_{k}\right\}$ que satisfaz

$$
F(z)=p^{-1}(z)[q(z)+H(z)]
$$

onde $H(z)=\mathcal{L}\left[\left\{B_{k}\right\}\right]$ e $s(z)=z^{n} D_{0}+\sum_{w=1}^{n-1} E_{w} z^{n-l}$ é uma $\lambda$-matriz de grau $n$ com $E_{w}=\sum_{i+j=w} A_{i} D_{j}$ e $A_{0}=I$.

Agora, vamos usar os resultados da seção anterior para determinar a seqüência. $\left\{Y_{k}\right\}$. A matriz $Y_{k}$ estará bem determinada se conhecermos seus elementos $y_{i j}^{(k)}$, para. $1 \leq i, j \leq m$. Como conseqüência do Teorema 5.1, podemos determinar a transformada inversa de Laurent (escalar) de cada $F_{i j}(z)$ como na Seçào 5.1, e assim obter as seqüências numéricas $\left\{y_{i j}^{(k)}\right\}$ tais que $F_{i j}(z)=\sum_{k=0}^{\infty} y_{i j}^{(k)} z^{-k}$.

No entanto, ao invés de calcular cada elemento $y_{i j}^{(k)}$ de $Y_{k}$, vamos proceder da. seguinte forma : primeiro, escrevemos $\left\{Y_{k}\right\}=\mathcal{L}^{-1}\left[p^{-1}(z) s(z)\right]+\mathcal{L}^{-1}\left[p^{-1}(z) H(z)\right]$.

Pelo Teorema 5.4 segue que se $z_{t}$ é um pólo distinto de $F_{i j}(z) z^{k-1}$ de ordem $\nu_{t}$ maior ou igual a 1 , então

$$
y_{i j}^{(k)}=\sum_{t=1}^{q} \operatorname{res}\left(F_{i j}(z) z^{k-1}, z_{t}\right) \text {, para } \quad 1 \leq q \leq m n,
$$

e, pela Observaçào 5.1,

$$
y_{i j}^{(k)}=\sum_{t=1}^{q} W_{i j}^{(1, t)} z_{t}^{k-1}
$$

ou seja, $\left\{Y_{k}^{-(1)}\right\}=\mathcal{L}^{-1}\left[p^{-1}(z) s(z)\right]$ é dada por

$$
Y_{k}^{(1)}=\sum_{t=1}^{q} W_{t}^{(1)} z_{t}^{k-1}, \quad k=0,1, \ldots,
$$

onde

$$
W_{t}^{(1)}=\frac{1}{\left(\nu_{t}-1\right) !} \frac{d^{\nu_{t}-1}}{d \lambda^{\nu_{t}-1}}\left[\frac{a(\lambda) s(\lambda)}{\prod_{\substack{w=1 \\ w \neq t}}^{q}\left(\lambda-\lambda_{w}\right)^{\nu_{w}}}\right]_{\lambda=\lambda_{t}} .
$$


Por outro lado, usando o teorema da convoluçào, $\mathcal{L}^{-1}\left[p^{-1}(z) H(z)\right]=Y_{k}^{(2)} * B_{k}$, onde $Y_{k}^{(2)}$ é determinado de modo análogo a $Y_{k}^{(1)}$ :

$$
Y_{k}^{(2)}=\sum_{t=1}^{q} W_{t}^{(2)} z_{t}^{k-1}
$$

para

$$
W_{t}^{(2)}=\frac{1}{\left(\nu_{t}-1\right) !} \frac{d^{\nu_{t}-1}}{d \lambda^{\nu_{t}-1}}\left[\frac{a(\lambda)}{\prod_{\substack{w=1 \\ w \neq t}}^{q}\left(\lambda-\lambda_{w}\right)^{\nu_{w}}}\right]_{\lambda=\lambda_{t}} .
$$

Concluímos que a solução $\left\{Y_{k}\right\}$ de (5.9) é dada por :

$$
\begin{aligned}
Y_{k}=\sum_{t=1}^{q} \frac{1}{\left(\nu_{t}-1\right) !} \frac{d^{\nu_{t}-1}}{d \lambda_{t}-1}\left[\frac{a(\lambda) s(\lambda)}{\prod_{\substack{w=1 \\
w \neq t}}^{q}\left(\lambda-\lambda_{w}\right)^{\nu_{u}}}\right]_{\lambda=\lambda_{t}} z_{t}^{k-1} \\
+\left\{\sum_{t=1}^{q} \frac{1}{\left(\nu_{t}-1\right) !} \frac{d^{\nu_{t}-1}}{d \lambda^{\nu_{t}-1}}\left[\frac{a(\lambda)}{\prod_{\substack{w=1 \\
w \neq t}}^{q}\left(\lambda-\lambda_{w}\right)^{\nu_{w}}}\right]_{\lambda=\lambda_{t}} z_{t}^{k-1}\right\} * U_{k} .
\end{aligned}
$$

No entanto, ainda devemos analisar a função $F_{i j}(z) z^{k-1}$ com mais atenção :

a) Se $z=0$ é um zero de $F_{i, j}(z)$, para $1 \leq i, j \leq m$, então (5.11) determina $\left\{Y_{k}\right\}$ para todo $k \geq 0$.

b) Se $z=0$ nào é nem pólo nem zero de $F_{i j}(z)$, para $1 \leq i, j \leq m$, então $(5.11)$ determina $\left\{Y_{k}\right\}$ para $k \geq 1$, já que $z=0$ é um pólo de $F_{i j}(z) z^{k-1}$ para $k=0$. $\mathrm{O}$ elemento $Y_{0}$ deve ser obtido separadamente, aplicando, por exemplo, o método numérico da transformada inversa ou aplicando o Teorema do Valor Inicial a cada. elemento $F_{i j}(z) z^{-1}$, conforme a Seção 5.1 .

c) Se $z=0$ é um pólo de ordem $\nu$ de $F_{i j}(z)$, para $1 \leq i, j \leq m$, então (5.11) determina $\left\{Y_{k}\right\}$ para $k \geq \nu+1$. Os elementos $Y_{0}, \ldots, Y_{\nu}$ devem ser calculados separadamente, como em (b). 


\subsection{Exemplo}

O exemplo de uma equação de diferença matricial linear que apresentamos nesta seção provém de uma equação diferencial matricial linear que descreve o movimento de uma nave espacial sem considerar agentes externos.

As equaçôes de movimento que governam uma nave espacial são as que governam um corpo rígido em rotação, e relacionam a posição angular com a velocidade angular. Se $E=\left\{e_{1}, e_{2}, e_{3}\right\}$ é um sistema de coordenadas ortonormal em $\mathbb{R}^{3}$, então a. posição angular da nave espacial é descrita por uma matriz de rotação $R$ que associa $E$ a um outro sistema de coordenadas ortonormal, cuja origem é o centro de massa da nave espacial. Denotamos por $w_{i}, i=1,2,3$, a velocidade angular da nave espacial em relaçâo ao eixo $r_{i}, i=1,2,3$, respectivamente. Nesse caso, $R e_{i}=r_{i}, i=1,2,3$.

A evolução de $R$ pode ser descrita por

$$
\dot{R}(t)=-R(t) S(w(t)),
$$

onde $w(t)=\left(w_{1}(t), w_{2}(t), w_{3}(t)\right)$ é a velocidade angular da nave espacial no tempo $t$ em relação aos eixos na nave espacial e a matriz $S(w(t)) \in \mathbb{C}^{3 \times 3}$ é dada por

$$
\left(\begin{array}{ccc}
0 & w_{3} & -w_{2} \\
-w_{3} & 0 & w_{1} \\
w_{2} & -w_{1} & 0
\end{array}\right) .
$$

Discretizando (5.12) pelo método de Euler, temos a seguinte equação de diferença. de primeira ordem :

$$
R_{t+1}-R_{t}\left(I-S\left(w_{t}\right)\right)=0 \text {. }
$$

Suponhamos que $w(t)$ é constante igual a $w$. Fazendo $I-S(w)=A$, a equação acima fica.

$$
R_{t+1}-R_{t} A=0
$$

cuja solução é dada por 


\subsection{Conclusão}

$$
R_{t}=R_{0}(I-S(w))^{t} .
$$

Observamos que uma equaçâo de diferença matricial linear de ordem maior pode ser obtida de (5.12), utilizando-se algum método de discretização de passos múltiplos.

\subsection{Conclusão}

Algumas consideraçòes devem ser feitas na escolha de um dos métodos apresentados neste trabalho para resoluçào de equações de diferença matricial linear.

No método do conjunto fundamental de soluçòes, a grande dificuldade está em determinar os solventes do polinômio característico matricial, isto no caso deles existirem. De posse dos solventes que satisfaçam o Teorema 3.1 ou o Teorema 3.2, um conjunto fundamental de soluçōes pode ser utilizado para gerar a soluçào geral $\left\{Y_{k}\right\}$ da equação de diferença matricial linear homogêna. Se a equação é não-homogênea, sua solução geral é a soma de $\left\{Y_{k}\right\}$ com uma solução particular da equação nãohomogênea. Um método de variação de constantes poderia ser desenvolvido como nos moldes das equaçòes de diferença escalares para determinar essa solução particular.

Já a cadeia de solventes permite obter a soluçào analítica do problema de Cauchy. A dificuldade, neste caso, é determinar a cadeia de solventes que, como vimos na Seçào 4.1, depende da fatorizaçào do polinômio característico em fatores lineares, sendo que, em geral, nem todo conjunto de solventes é uma cadeia de solventes de um polinômio matricial. Uma generalizaçâo de cadeia de solventes, a cadeia de co-solventes, também pode ser utilizada para resolver o problema de Cauchy [17].

E, por fim, com a aplicaçào da transformada de Laurent a uma equaçào de diferença matricial linear nâo-homogênea, não necessitamos de solventes, e sim de raízes de um polinômio escalar (raízes latentes de uma lambda-matriz). A determinação da matriz adjunta de uma lambda-matriz e de suas derivadas pode ser feita. 
numericamente .

Dessa forma, se não conhecemos os solventes do polinômio característico ou se este não admitir uma cadeia de solventes, parece-nos que o último método é o mais indicado para determinar a solução de uma equação de diferença matricial linear com condições iniciais. 


\section{Bibliografia}

[1] J.B. Conway. Function of one complex variable. Springer, 1978.

[2] J. Dennis, J. Traub, and R. Weber. The algebraic theory of matrix polynomials. SIAM J. Numer. Anal., 13(6):\$31-\$45, 1976.

[3] W. J. Frazer, R. A.; Duncan and A. R. Collar. Elementary matrices and some applications to dynamics and differential equations. University Press, Cambridge, 1955.

[4] F. R. Gantmacher. The theory of matrices. Chelsea Pub. Co., New York, 1960.

[5] I. Gohberg, P. Lancaster, and L. Rodman. Matrix polynomials. Academic Press, Inc., 1982.

[6] S.L. Goldberg. Introduction to difference equation: with illustrative examples from economics, psychology and sociology. John Wiley, 1958.

[7] V. Hernandez and F. Incertis. A block bidiagonal form for block companion matrices. Linear Algebra and Its Applications, 75:241-256, 1986.

[8] L. Jódar and E. Navarro. On complete set of solutions for polynomial matrix equations. Appl. Math. Lett., 3(1):15-18, 1990.

[9] E.I. Jury. Theory and applications of z-transformation method. Willey, New York, 1964.

[10] J. Kalman. Generalized vandermonde matrices. Math. Mag., 57:15-21, 1984. 
[11] W.G. Kelley and A.C. Peterson. Difference equations - An introduction with applications. Academic Press Inc., 1991.

[12] P. Lancaster. Lambda-matrices and vibrating systems. Perganon Press, 1966.

[13] D.F. Lawden. On the solution of linear difference equations. Math. Gazette, 36:193-196, 1952.

[14] M. Marcus and H. Minc. A survey of matrix theory and matrix inequalities. Allyn and Bacon, Inc., 1964.

[15] M. Markus and M. Mereutsa. On the complete n-tuple of roots of operator equations. Math. USSR Izv., 7:1105-1128, 1973.

[16] J. Maroulas. Factorization of matrix polynomials with multiple roots. Lin. Alg. Appl., 69:9-32, 1985.

[17] F.E. Menzaque. Equações polinomiais matriciais: métodos de resolução. Tese de Doutorado - IME-USP - 1993.

[18] K. Miller. Linear difference equation. W. A. Benjamin Inc., 1968.

[19] R. Vich. The Z-transformation theory and its applications. D. Reidel Pub. Co., 1987. 